
\title{
3 Research Square \\ The differential expression patterns of paralogs in response to stresses indicate expression and sequence divergences
}

Shuaibin Lian ( $\nabla$ shuai_lian@xynu.edu.cn )

Xinyang Normal University https://orcid.org/0000-0002-0211-1842

\section{Yongjie Zhou}

Xinyang Normal University

Zixiao Liu

Xinyang Normal University

Andong Gong

Xinyang Normal University

\section{Lin Cheng}

Xinyang Normal University

Research article

Keywords:

Posted Date: October 14th, 2019

DOI: https://doi.org/10.21203/rs.2.16022/v1

License: (9) This work is licensed under a Creative Commons Attribution 4.0 International License. Read Full License

Version of Record: A version of this preprint was published on June 16th, 2020. See the published version at https://doi.org/10.1186/s12870-020-02460-x. 


\section{Abstract}

Background Theoretically, paralogous genes generated through whole genome duplications should share identical expression levels due to their identical sequences and chromatin environments. However, functional divergences and expression differences have arisen due to selective pressures and evolutional stresses. A comprehensive investigation of the expression patterns of paralogous gene pairs in response to various stresses and a study of correlations between the expression levels and sequence divergences of the paralogs are needed.Results In this study, we analyzed the expression patterns of paralogous genes under different types of stress and investigated the correlations between the expression levels and sequence divergences of the paralogs. Firstly, we analyzed the differential expression patterns of the paralogs under four different types of stress (drought, cold, infection, and herbivory) and classified them into three types according to their expression patterns. Secondly, we further analyzed the differential expression patterns under various degrees of stress and constructed the corresponding co-expression networks of differentially expressed paralogs and transcription factors. Thirdly, we investigated the correlations between the expression levels and sequence divergences of the paralogs and identified positive correlations between expression level and sequence divergence. With regard to the sequence divergence, we identified correlations between selective pressure and phylogenetic relationship.Conclusions These results shed light on differential expression patterns of paralogs in response to environmental stresses and are helpful for understanding the relations between expression levels and sequences divergences.

\section{Background}

Several studies have reported that most plants have undergone multiple rounds of whole genome duplication (WGD) [1-3], which has long been recognized as an important evolutionary force. At least one ancient WGD occurred before the divergence of monocots and eudicots in angiosperm evolution. For example, Arabidopsis thaliana has undergone two recent WGD events, with the most recent one occurring at approximately 23 million years ago (Mya) [4]. Soybean (Glycine max) has also experienced two WGDs [5], which occurred at approximately 13 Mya (the most recent WGD) and 59 Mya. WGDs can duplicate chromosomes, thereby resulting in a large number of duplicate genes. These duplicate genes are considered to play important roles in enhancing organisms' adaptation to the environment and promoting species diversification [6-9]. The functions of the duplicate genes have diverged remarkably throughout evolution, although most duplicate genes have been lost [10,11].

Although many mechanisms can explain the functional divergences of the duplicates, the paralogous genes generated through WGDs should initially share identical sequences and chromatin environments, and possess stronger expression correlations than the other duplication types [12]. Theoretically, the paralogs should share identical expression levels in the absence of selective pressures and stress [13], because they share identical sequences. Functional divergences and expression differences have arisen due to selective pressures and inherently harsh environments after hundreds of millions of years of evolution [14]. The divergences in the regulatory regions of genes may have changed their expression 
patterns, whereas changes in the coding regions may have resulted in the acquisition of new functions [15-17]. Therefore, gene expression divergence is an important evolutionary driving force for paralogs.

Several studies have examined the relationship between the sequence and expression divergence of duplicates [17-21]. Warnefors and Kaessmann investigated the correlations between the divergence of gene and protein expression in mammals and identified several positive correlations [22]. However, a study in sunflower has indicated that there are no correlations; instead, this study has described decoupling between gene expression and sequence divergence [23]. Similar results were reported in the flycatcher species [24]. Furthermore, many studies have confirmed that genes with high expression levels evolve more slowly than those with low expression levels (for review, [25]) and reported the correlations between expression divergence and selective pressure. For example, studies in Drosopila implicated that positive selection is closely related to expression divergence [26], whereas others have reported contradictory findings, that is, purifying selection is the primary driving force of the divergences in expression and sequence [27]. Consequently, it is interesting to ask whether there are correlations between expression divergences of paralogs and selective pressures or stresses in plants.

Thus, we investigated the differential expression patterns and expression divergences of paralogs under four different types of stress (two biotics stresses and two abiotic stresses) in Arabidopsis thaliana. Furthermore, we identified the correlations between sequence divergences and selective pressures. Lastly, we constructed the co-expression networks of paralogs with different expression patterns and associated transcription factors.

\section{Results}

\section{Homolog identification and paralog expression classification}

We identified 7789 paralogs (paralogous gene pairs) in the model plant species Arabidopsis thaliana based on a homology analysis involved 20 other species using the Inparonoid 7 Software (Methods and Materials) [28]. The list of 7789 paralogs was presented in Table. S1. The phylogenetic relationships of the 21 species was obtained from Lian et al. and Ren et al. [2, 29]. Thereafter, we analyzed the interactions and distributions of the paralogs and repeats in the chromosomes, respectively (Fig. 1A). The corresponding interactions information were presented in Table. S2 and Table. S3. The repeats of Arabidopsis thaliana were identified using the RepeatMasker and HashRepeatFinder tools (Methods and Materials). These results indicated that the paralogs and repeats were highly coincident with regard to their locations and interactions, and the corresponding coincidence rate was $82.4 \%$, which further confirmed that the paralogous gene pairs were mostly generated through genome duplications, including WGDs and small-scale duplications (SSDs) [2,30].

Thereafter, we classified the paralogs into three types (FF, FP or PP) (see definitions in Methods and Materials) according to their expression patterns under four different types of stress, including two biotic stresses (infection by the necrotrophic fungus Botrytis cinerea, $B C$, and herbivory by the chewing larvae of Pieris rapae, $\mathrm{Pr}$ ) and two abiotic stresses (drought, Dr and cold, $\mathrm{Cd}$ ). By differential expression analysis, 
we identified 436, 1723, and 5630 pairs of FF, FP, and PP paralogs in Drstress; 444, 1865, and 5480 pairs of FF, FP, and PP paralogs in Cd stress; 713,1976 , and 5100 pairs of FF, FP, and PP paralogs in $B c$ stress; and 161, 850, and 6778 pairs of FF, FP, and PP paralogs in Prstress, respectively (Fig. 1B). The corresponding proportions of FF, FP, and PP were $5.6 \%, 22.1 \%$, and $72.3 \%$ in Drstress; $5.7 \%, 23.9 \%$, and 70.3\% in Cd stress; $9.2 \%, 25.3 \%$, and $65.5 \%$ in Bc stress; and 2.1\%, $10.9 \%$, and $87 \%$ in Prstress, respectively. The gene list of FF, FP and PP under four different stresses was presented in Table. S4. The statistic significances of differential expression of FF and FP under the four different types of stress were examined by using Mann-Whitney U-test (Fig. 1B). Significance value was indicated with $P<0.05$. The $\log 2|\mathrm{FC}|$ values of FF and FP paralogs under four different stresses were presented in Table. S5. We also investigated the co-expressed FF paralogs under the four different types of stress by computing Pearson coefficient $r$. The proportions of the co-expressed FF paralogs were $75 \%$ in $D r$ stress, $84 \%$ in $C d$ stress, 78.5\% in Bc stress, and 91.9\% in Prstress, respectively (Fig. 1B). The threshold of Pearson coefficient is $r>0.5$.

These results showed that (1) most paralogous genes were not expressed or differentially expressed, and only a small proportion of the paralogous genes were both differentially expressed, which suggests that most paralogous genes are not involved in stress response mechanisms; and (2) the expression patterns of paralogs involved in stress response were significantly different, especially for FF and FP paralogs, which suggests that these paralogs (DEPs) are significantly differentially expressed in stress response; (3) most paralogs with FF expression patterns under four different types of environmental stress tend to show similar expression pattern.

\section{Differential expression patterns of paralogs under biotic and abiotic stress}

To investigate the differential expression patterns of FF and FP paralogs under the four different types of stress, we analyzed the FF and FP paralogs under different types of stress and generated a Venn diagram (Fig. 2 for FF, Fig. S1 for FP). We clustered all 1189 FF paralogs and 3259 FP paralogs into seven expression modules according to their differential expression patterns under different types of stress. The $\log 2|\mathrm{FC}|$ values of seven FF and FP expression clusters were presented in Table. S6 and Table. S7. The corresponding heatmaps and specific functions of the FF and FP paralogs were presented in Fig. 2 and Fig. S1, respectively. Furthermore, we identified the TFs in each cluster. The FF and FP paralogs belonging

to the first three clusters were differentially expressed with regard to the four different types of stress. The paralogs belonging to the last four clusters were differentially expressed with regard to only one type of stress. We performed function enrichment and KEGG analysis for the FF paralogs to assign functional categories to each module (Fig. 2D).

With regard to clusters 1 through 3, cluster 1 contained five DEPs, two of which were TFs and were shared by all four types of stress (Fig. 2A-C). Functional enrichment analysis indicated that these five paralogs were mainly involved in galactose metabolism, and two TFs were $b H L H$ transcription factors. These results indicate that plants require more energy to deal with harsh environments, which have been confirmed by a recent study [31]. Cluster 2 contained 91 differentially expressed paralogs, eighteen of 
which were TFs and were shared by abiotic stresses (Drand Cd) (Fig. 2A-C). The functions of DEPs in cluster 2 were mainly those involved in the response to various abiotic stresses, such as water deprivation, temperature fluctuations, and karrikin (Fig. 2D). Studies have confirmed that these genes are involved in the biosynthesis of abscisic acid, and they improved the abiotic stress tolerance in Arabidopsis thaliana [32, 33]. Karrikin, a signaling molecule, is found in smoke from burning vegetation, and it triggers seed germination for many angiosperms (i.e., flowering plants) [34]. This may be a protective mechanism used by plants for seed development in response to harsh environmental conditions, such as drought, cold, and high salinity [35]. Cluster 3 contained 33 differentially expressed paralogs, eleven of which were TFs and shared by biotic stresses (Fig. 2A-C). The corresponding functions were mainly involved in the response to various biotic stresses, such as protection from attacks by fungi, bacteria, and oomycetes as well as immunological processes. We identified five WRKYTFs (i.e., WRKY6, WRKY40, WRKY54, WRKY70 and WRKY18), thereby reflecting the important roles of WRKYTFs in the response to biotic stress. For example, WRKY70 and WRK54 are involved in basal defense mechanisms against Hyaloperonospora parasitica and disease resistance in Arabidopsis [36]. On the other hand, WRKY 6 and WRKY4O play important roles in transducing E-2-hexenal perception, which is a green leaf volatile (GLV) that is produced upon wounding, herbivory or infection by pathogens [37].

With regard to clusters 4 through 7, we identified 216 (containing 33 TFs), 269 (containing 38 TFs), 449 (containing $87 \mathrm{TFs}$ ), and 66 paralogs (containing $25 \mathrm{TFs}$ ) that were differentially expressed under $\mathrm{Dr}, \mathrm{Cd}$, $B C$, and Prstress, respectively. The proportions of the co-expressed genes in one paralog were $7.4 \%, 7.8 \%$, $9.8 \%$, and $21.2 \%$, respectively (Fig. 2A-C). The functional enrichment of cluster 4 indicated that 261 paralogs were mainly enriched in carbohydrate biosynthesis, photosynthesis, and drought recovery. Furthermore, $b H L H$ negatively regulates jasmonate signaling and improves the tolerance to drought stress [38]. The functions of cluster 5 were mainly enriched in the response to cold and UVs. As previously reported, these genes are involved in diurnal oscillation and beta-amylase biosynthesis, which increases the sensitivity of PSII photochemical reaction to freezing and ambient stress in Arabidopsis [39, 40]. The functions of clusters 6 and 7 were mainly enriched in systemic resistance, toxin metabolism, immune response, and protection from insects (Fig. 2D).

These results indicate that (1) paralogs with different expression clusters participate in different biological processes and have different biological functions; (2) the paralogous genes with functional redundancy were differentially expressed during the exposure to different types of stress, and (3) the expression patterns of the paralogous genes can change under different stress conditions.

\section{Differential expression patterns of paralogs under different degrees of the same type of stress}

We investigated the effects of different degrees of stress on the expression patterns of the paralogs and classified the paralogs into two types according to expression level, which we defined as the enhancing expression pattern () and decreasing expression pattern () (Fig. 3). We identified 1721 and 147, 2037 and 118, 2262 and 201, and 442 and 468, enhancing and decreasing paralogs in $D r, C d, B c$, and Prstress, 
respectively (Fig. 3A, B). The log2|FC| values of paralogs in enhancing and decreasing patterns under four stresses was presented in Table. $\mathbf{8 8}$ and Table. $\mathbf{S 9 .}$

For the enhancing expression pattern, the paralogs were not expressed or differentially expressed at the onset of different stress. With prolonged or increased stress, more paralogs became differentially expressed (Fig. $3 \mathrm{~A}, \mathrm{C}$ ). At the weakest phase of $\mathrm{Dr}, \mathrm{Cd}, \mathrm{BC}$, and Prstress, the proportions of DEPS were $4.9 \%, 2.4 \%, 3 \%$, and $6.3 \%$, respectively. At the middle phase, the proprtions of DEPs were $36.8 \%, 28.8 \%$, $14.6 \%$, and $35.5 \%$, respectively. At the strongest phase, the proportions of DEPs all reached $100 \%$. The functional enrichment of the paralogs indicated that those responsive to the Drstress were mainly involved in processes related to water deprivation and photosynthesis [41], those responsive to the $C d$ stress were mainly involved in processes related to temperature fluctuations and cold [42], those responsive to the $B c$ stress were mainly involved in processes related to protection from bacterial infection [43], and those responsive to the Prstress were mainly involved in processes related to the defense response and immunological events [44]. Furthermore, we found that most enhancing paralogs were differentially expressed in at least two different types of stress simultaneously, and the proportions of the up-regulated paralogs in $D r, C d, B C$, and $P r$ co-enhanced with another type of stress were $68.7 \%$, $60.3 \%, 57 \%$, and $41.2 \%$, respectively (Fig. $3 \mathrm{C}$ ). These results indicate that most paralogs can respond to or be activated by several types of stress. The functional enrichment analysis of 353 paralogs that responded to both $\mathrm{Dr}$ and $C d$ stress confirmed the functional redundancy with regard to water deprivation and temperature fluctuations. The functions of 25 paralogs (Fig. 3A) shared by the four types of stress were mainly enriched in ion homeostasis and auxin transport [45], which have been reported to be involved in most fundamental events [46, 47].

For the decreasing expression pattern, the paralogs were significantly differentially expressed at the onset of different types of stress. With prolonged stress, more paralogs were not expressed or differentially expressed (Fig. 3B, D). At the weakest phase of $D r, C d, B c$, and Prstress, the proportions of DEPs were all $100 \%$. At the middle phase, the proportions of DEPs were $24.4 \%, 51.7 \%, 38.8 \%$, and $34.8 \%$, respectively. At the strongest phase, the proportions of DEPs were $7.5 \%, 7.6 \%, 7.4 \%$, and $6 \%$, respectively. The functional enrichment of the paralogs indicated that those responsive to $\mathrm{Dr}$ and $C d$ stress were mainly involved in processes related to monocarboxylic acid and carboxylic acid biosynthesis. Recent studies have reported that these small molecules can help plants to adapt to extreme stress conditions $[48,49]$.

These results indicate that the expression patterns of the paralogs vary under different types of stress as well as with different degrees of stress, suggesting that the expression levels of paralogs is not only related to the type but also the severity of stress. These results also reveal that most paralogs are differentially expressed in response to multiple stresses, probably suggesting that the functional redundancy of paralogs is a protective mechanism for the adaptation of plants to different stress environments throughout evolution.

\section{Co-expression networks of DE paralogs and transcriptional factors under different types of stress}


To understand how transcription factors (TFs) regulate DEPs expression in response to stress, we constructed their co-expression networks for $D r, C d, B c$, and Prstresses (Fig. 4).

The co-expression networks revealed several important insights. Firstly, the proportions of down-regulated DEPs in enhancing and decreasing expression patterns under $D r, C d, B C$, and $\operatorname{Prstress}$ were $100 \%$ and $36.7 \%, 93.3 \%$ and $90 \%, 96.7 \%$ and $86.7 \%$, and $100 \%$ and $100 \%$, respectively. These results indicate that DEPs with both enhancing and decreasing patterns showed low expression, except for DEPs with a decreasing pattern under $D r$ stress. Secondly, the top three TFs co-expressed with DEPs were MYB, ERF, and $b H L H$ under Drstress (Fig. 4A); $E R F, b H L H$, and NAC under $C d$ stress (Fig. 4B); ERF, MYB, and WRKY under $B C$ stress (Fig. 4C); and ERF, NAC, and MYB under Prstress (Fig. 4D). The corresponding proportions were $14 \%, 13 \%$, and $12 \%$ under Drstress; $19 \%, 9 \%$, and $9 \%$ under $C d$ stress; $19 \%, 14 \%$, and $10 \%$ under $B c$ stress; and $17 \%, 15 \%$, and $12 \%$ under Prstress. Previous studies have reported that ERF plays important roles in the responses to both biotic and abiotic stresses [50-52]. For example, ERF9 protects Arabidopsis from necrotrophic fungi, and post-anaerobic reoxygenation-the main defense mechanism in plants [53]-is regulated by ERF96 [54]. A study has also confirmed that $b H L H$ can mediate the trade-off between abiotic and biotic molecular pattern-triggered immunity in Arabidopsis [55, 56]. However, MYB is mainly involved in the response to biotic stress $[57,58]$. Thirdly, we identified specific TFs under different types of stress. For example, Nin-like is a master regulator of the response of Arabidopsis to Drstress [59]. E2FD/DEL2 controls cell proliferation in Arabidopsis during exposure to $C d$ stress [60]. BES1 promotes brassinosteroid signaling and development in Arabidopsis thaliana during exposure to $B c$ stress [61]. Finally, there were more interactions between DEPs and TFs with an enhancing expression pattern than those with a decreasing expression pattern, which was applicable to $\mathrm{BC}$ and $\mathrm{Pr}$ stress with corresponding excess ratios of $73.8 \%$ and $79.4 \%$, respectively (Fig. 4C, D). An increased number of interactions indicated that more TFs regulated the responses of the paralogs to the enhancing severity of stress. These results are very helpful for understanding the regulatory mechanisms of TFs with regard to the responses of paralogs to stress.

\section{Expression divergences positively correlate with sequence divergences}

We continued our study by investigating whether there were positive or negative correlations between expression divergences and sequence divergences [62]. The paralogs with FF and FP expression patterns were investigated. To estimate the sequence divergence between paralogs, we computed the synonymous $(K s)$ substitution rate, which is recognized as a proxy of the sequence divergence time. According to previous studies [21, 62], we used the rescaled Pearson's correlation coefficient to perform linear regression analysis (Methods and materials). The regression results of the expression levels of FF and FP paralogs and the $K s$ rates are presented in Fig. $\mathbf{5}$.

We found a significant negative correlation between the rescaled and $K s$ values for FF and FP gene pairs $(P<0.001$, U-test, Fig. 5A). The negative correlation between the and $K s$ values was indicative of a positive correlation between expression divergence and sequence divergence. These results indicate that the expression divergences of both FF and FP gene pairs were positively correlated with sequence 
divergences. Furthermore, we investigated the distribution of $K s$ values for $\mathrm{FF}$ and $\mathrm{FP}$ paralogs and identified one peak with a value of 1.8 in the density plot (Fig. 5B). These results indicate that the gene pairs originating at the value of 1.8 experienced a large amount of synonymous substitution. More than $80 \%$ of FF and FP paralogs had $K s$ values larger than 1.0 , suggesting that they have persisted for a relatively long evolutionary time and are highly diverged. In addition, the gene pairs near the $K s$ peak probably experienced larger expression divergences [63].

We also investigated the correlations of DEPs with enhancing and decreasing expression patterns under $D r, C d, B c$, and $P r$ stress. We identified a negative correlation between the expression divergences and $K s$ value for all four types of stress $(P<0.001$, U-test, Fig. 5 C). These results indicate that the expression divergences of DEPs in response to stress were positively correlated with sequence divergences. Furthermore, the density plot of the corresponding $K a$ and $K s$ values implied a $K s$ peak value of 1.8 (Fig. 5D), indicating that these genes have persisted for a relatively long evolutionary time and are highly diverged.

In summary, this study reveals new correlations between the expression divergences and sequence divergences of paralogous genes, which can help us understand the evolutionary mechanism of stress adaptation in plants.

\section{Selective pressures support the expression divergences of the paralogs}

We investigated whether there were correlations between expression divergences and selective pressures. To infer selective pressures, we used FF and FP DEPs under $D r, C d, B c$, and Prstress to compute their nonsynonymous/synonymous substitutions rate ratios $(K a / K s)$. The boxplot of $K a$ and $K s$ values, as well as the $K a / K s$ ratios, of FF and FP DEPs under the four types of stress are presented in Fig. 6.

These results revealed two important insights. Fistly, the median value of the $K a / K s$ ratio for FP was consistently larger than 1.0, but that of FF was smaller than 1.0 for all four types of stress, indicating that the FP gene pairs underwent positive selection but the FF gene pairs underwent purifying/negative selection. Secondly, the $K a$ and $K s$ values of FP for all four types of stress were consistently larger than those of FF, revealing that the FP gene pairs experienced more non-synonymous/synonymous substitutions and were evolutionarily older than the FF gene pairs. To ensure that the phenomena we observed were not due to chance, we compared our results with a randomized experiment containing an equal number of randomized gene pairs (Fig. S2, Methods and materials), and found that the $K a / K s$ ratio of FF was consistently smaller than 1.0 and that of the randomized experiment [29], but the $K a / K s$ ratio of FP was consistently larger than 1.0 and that of the randomized experiment $(P<)$. Statistical significance was indicated by 10,000 times of the randomized experiment.

These results indicate that FF paralogous pairs experienced relaxed selection constraints and retained functional redundancy, but FP paralogous pairs experienced strong positive selection and more sequence divergence, which led to function divergence. These findings suggest that paralogs with different expression pattern probably experienced different selection constraints. 


\section{Discussion}

\section{Sequence divergences of the paralogs support the phylogenetic relationships among species}

To investigate the correlations between sequence divergences and phylogenetic relationships, we examined the synonymous substitution rate (Ks) of paralogs between Arabidopsis thaliana and 20 other species (Fig. 7A). The corresponding boxplot of $K s$ values is presented in Fig. 7B. Generally, smaller $K s$ values indicated less synonymous substitutions and divergences as well as stronger phylogenetic relationships. The results in Fig. 7 show that three species, Arabidopsis lyrata, Boechera stricta, and Brassica rapa, had much smaller $K s$ values $(0.3707,0.878$, and 0.905 , respectively) for Arabidopsis thaliana, as compared with 17 other species (all larger than 1.0), indicating that the genomes of these three species display less divergence and closer phylogenetic relationships with Arabidopsis thaliana, which is consistent with the phylogenetic results of angiosperms [64]. Furthermore, we investigated the relationship between species conservation and family size and identified an inversely proportional correlation between species conservation and family size (Fig. S3). The family size of the paralogs significantly decreased as the occurrence of the species increased. A recent study has proposed a model of exponential decrease of duplicate genes over time [2]. Further studies are needed to investigate whether the relationship between species conservation and family size of the paralogs fits the exponential decay model, as these results may improve our understanding of the evolution of the duplicate genes.

\section{Conserved domains and cis-elements}

A recent report has confirmed that the expression divergence of the duplicated genes is attributed to alterations in the cis-elements [65], which have been proposed to mediate the expression divergence of genes in rice [66]. Thus, we further investigated the conserved domains and cis-elements of the paralogs in all 21 species.

We identified one paralogous gene family with seven genes in all 21 species. We used the CDD Database to identify their conserved domains. The most highly conserved protein domain was the catalytic domain of the serine/threonine kinases (STKs), interleukin-1 receptor associated kinases and related STKs (STKcIRAK) (Fig. 8). The STKs catalyze the transfer of the gamma-phosphoryl group from ATP to serine/threonine residues on the protein substrates. IRAKs are involved in the Toll-like receptor (TLR) and interleukin-1 (IL-1) signaling pathways. Thus, they regulate innate immune responses and inflammation $[67,68]$. Using the MEME Software, we identified 15 conserved motifs of STKc-IRAK, and found that most motifs were widespread in TFs, such as $\angle B D, A R F, S A P$, Whirly, SRS, Dof, and GRAS (Fig. 9A, B). Furthermore, the seven genes in all 21 species shared similar motif structures and gene lengths.

We used PlantCARE to predict cis-element variations of the STKc-IRAK gene family and identified 13 cicelements related to stress in the 2000-bp promoter sequence of the paralogous gene family (Fig. 10). The top ten components are shown in Fig. 10A, and they include the low temperature response component $(L T R), M Y B$ binding site involved in the drought-induced component (MBS), MeJA reaction component 
(CGTCA-motif), salicylic acid reaction component (TCA-element), gibberellin reaction component (GAREmotif and $P$-box), auxin response element (TGA-element), abscisic acid reaction component ( $A B R E)$, MeJA element (TGACG-motif), stress response element (TC-rich repeats), and optical response elements (3-AF1 binding site, GT1-motif, and Sp1). The number of cis-elements identified in each gene is shown in Fig. 10B. Among them, the top two elements were the CGTCA-motif and TGACG-motif, accounting for $25 \%$ for all elements. These cis-elements are all related to stress, which suggests that they may be involved in the transcriptional control of abiotic stresses and hormonal responses [69].

\section{Conclusions}

In this study, we analyzed the expression patterns of paralogous genes under different types of stress and investigated the correlations between the divergences in expression and sequence of the paralogs. Firstly, we analyzed the differential expression patterns of the paralogs under four different stresses ( $D r$, $C d, B C$, and $P r)$ and classified them into three types according to their expression pattern. Secondly, we further analyzed their differential expression patterns under different degrees of stress and constructed the corresponding co-expression networks of differentially expressed paralogs and TFs. Thirdly, we investigated the correlations between the divergences in expression and sequence and identified positive correlations between the expression divergences and sequence divergences. Lastly, we found that paralogs with different expression patterns probably experienced different selection constraints and FF paralogous pairs experienced relaxed selection constraints but FP paralogous pairs experienced strong positive selection. These results probably suggest that paralogs experienced relaxed selection tend to be functional redundancy but those experienced strong positive selection tend to show more sequence divergence, which provide new insights for understanding the differential expression patterns of paralogs in response to environmental stresses and sequence divergences.

\section{Methods}

\section{Homolog identification and paralog classification}

We used the homolog analysis software InParanoid 8 with default parameters to identify paralogous gene pairs between Arabidopsis thaliana and 20 other species according to their phylogenetic relationships (Fig. 7A) [28]. After removing the identical gene pairs, 7,789 paralogous gene pairs (paralogs) remained. Thereafter, we classified each paralogous gene pair into one of three types (FF, FP or PP) according to whether it was differentially expressed under different stress conditions. FF paralogs refer to paralogous gene pairs that both genes in a pair were differentially expressed. FP paralogs refer to paralogous gene pairs that one gene in a pair was differentially expressed and the other was not expressed or differentially expressed. PP paralogs refer to paralogous gene pairs that both genes in a pair were not expressed or differentially expressed.

\section{Transcriptome analysis}


The transcriptome data of Arabidopsis thaliana under drought stress, cold stress, infection by the necrotrophic fungus Botrytis cinerea, and herbivory by the chewing larvae of Pieris rapae were obtained from the Chinese Academy of Sciences with Bio-Project Accession No. PRJNA525452 (https://www.ncbi.nlm.nih.gov/bioproject/525452) [70]. We firstly used Trimmomatic-0.36 software to remove the low-quality RNA-sequencing reads, and then used Hisat 2-2.0.4 to map clean reads to reference genomes with default parameter settings and generate bam files. Lastly Cufflinks (V2.2.0) software was used to generate FPKM values of gene expression levels, and then we used the edgeR tools to identify differentially expressed genes (DEGs) under four different types of stress with parameters padj $<0.05$ and $|\log 2 \mathrm{FC}|>1$, respectively [71]. For differential expression pattern, we used transcriptome data at $7 \mathrm{~d}$ for $D r$ stress, $24 \mathrm{~h}$ for $C d$ stress, $18 \mathrm{~h}$ for Bc stress and $18 \mathrm{~h}$ for Prstress. For enhancing and decreasing expression pattern, we used transcriptome data at 5d, 6d, 7d for Drstress, 3h, 6h, 24h for $C d$ stress, 6h, 12h ,18h for Bc stress, 6h, 12h, 18h for Prstress.

\section{Interactions and distribution analysis}

We used the RepeatMasker and HashRepeatFinder tools to identify repetitive sequences in Arabidopsis thaliana. The threshold of similar repetitive sequences was set to $85 \%$, and repeats shorter than 150 nucleotides were removed. We determined the locations of the repeats and paralogs on the chromosomes using the annotation data and used the GlobalOptions and Circlize modules in R Package to identify their interactions and distributions on the chromosomes.

\section{Weighted gene co-expression network analysis}

The weighted gene co-expression network analysis (WGCNA) tool within R Package summarizes and standardizes the methods and functions for co-expression network analysis [72]. The WGCNA network construction tool was used to generate the nodes and edges of the genes by computing the correlations of the expression values. The nodes corresponded to genes, and the edges were determined by pairwise correlations between gene expression levels. The corresponding calling function within R Package was 'blockwiseModules'. The parameters were set as follows: powers $=10$, minModuleSize $=30$, and mergeCutHeight $=0.25$, and other parameters were set to their default settings. The nodes with a correlation of $r 0.5$ and edges with a weighted threshold of $<0.3$ were removed. Thereafter, the Cytoscape tool (https://cytoscape.org/) was used to plot the interactions using the nodes and edges of conserved genes.

\section{Expression and sequence divergence analysis}

The non-synonymous $(K a)$ and synonymous $(K s)$ substitutions of each paralog were computed using the 'dnds' function within MATLAB. $K a / K s>1$ indicates that the gene experienced positive selection, $K a / K s<1$ indicates that the gene experienced negative selection, and $K a / K s=1$ indicates that the gene experienced neutral evolution [73]. The boxplots of $K a$ and $K s$ values were generated using the 'ggplot2 function within R Package. The Pearson coefficient of the expression level of each paralog gene pair was computed using the 'corr' function within MATLAB using the following equation: 


$$
r=\frac{\sum X Y-\frac{1}{N} \sum Y \sum Y}{\sqrt{\left(\sum X^{2}-\frac{1}{N}\left(\sum X\right)^{2}\right)\left(\sum Y^{2}-\frac{1}{N}\left(\sum Y\right)^{2}\right)}}
$$

where $X, Y$ represent the expression data of the two genes at different time points.

Expression divergence was measured using the rescaled Pearson coefficient $[36,62]$, which is more appropriate for linear regression analysis.

$$
r^{\prime}=\frac{\ln (1+r)}{1-r}
$$

Linear regression analysis was performed using the ' $I m$ ' function within R Package, with the rescaled; the negative regression coefficient between and $K s$ (or $K a$ ) represents positive relationship between expression level and $K s$ (or Ka) value.

\section{Randomized experiments}

We simulated randomized experiments to test the statistical significance of $K a$ and $K s$ for the FF and FP paralogs [29]. When the selective pressure was not characteristic of the FF and FP gene pairs, the results of the randomized experiment and real data were similar. To achieve this, we randomly generated an equal number of FF and FP gene pairs for each stress condition from 7,789 paralogs. We repeated the randomized experiment 10,000 times to evaluate the intrachromosomal colocalization of these random pairs. For example, to test the significance of the $K s$ value for $436 \mathrm{FF}$ paralogs under $D r$ stress, we randomly generated 436 gene pairs from the 7,789 paralogs, and computed their $K s$ values; this process was repeated 10,000 times. The frequency distributions of the $K a$ and $K s$ rates, as well as the $K a / K s$ ratio, with 0.1 steps are shown in Fig. $\mathbf{2}$.

\section{Statistical methods}

Mann-Whitney U-test (function 'ranksum' in software'MATLAB' version R2016b) was used to examine the statistical significance between given two samples, the default significance level is 0.05 . The MannWhitney U-test is a nonparametric test for equality of population medians of two independent samples. The main advantage of this test is that it makes no assumption that the samples are from normal distributions.

\section{Cis-element and conserved domain analysis}

The online platform PlantCARE (http://bioinformatics.psb.ugent.be/webtools/plantcare/html) was used for cis-element analysis utilizing the 2,000 bp promoter regions of the seven paralogs [74]. The Multiple Em for Motif Elicitation (MEME) software (http://meme-suite.org/tools/meme) was used for motif discovery. The parameters were as follows: the motif number was 15 , and the motif width was 50 amino acids. The Conserved Domain Database (CCD, https://www.ncbi.nlm.nih.gov/cdd/) was used to analyze 
the conserved domain sequences [75]. Functional enrichment was performed by using Metascape tools [76], resulting $P$ values were adjusted to $Q$ values by the Benjamini-Hochberg correction, and a false discovery rate of $5 \%$ was applied.

\section{Availability of data and materials}

The genetic data of the 21 species listed in Fig. 7A, including the CDS sequences and annotation data, were downloaded from the EnsemblPlants Database (http://plants.ensembl.org/info/website/ftp/index.html). In addition, 2,296 transcription factors (1,717 loci) of Arabidopsis thaliana were downloaded from the Plant Transcription Factor Database (http://planttfdb.cbi.pku.edu.cn/index.php).

\section{Declarations}

\section{Ethics approval and consent to participate}

Not applicable

\section{Consent for publication}

Not applicable.

\section{Supplementary data}

Table. S1. The gene list of 7789 paralogs.

Table. S2. The interaction information of paralogs.

Table. S3. The interaction information of repeats.

Table. S4. The list of FF, FP and PP paralogs under four different stresses.

Table. S5. The log2FC values of FF and FP paralogs under four different stresses.

Table. S6. The log2FC values of seven FF paralogs expression clusters.

Table. S7. The log2FC values of seven FP paralogs expression clusters.

Table. S8. The log2FC values of paralogs in enhancing patterns under four stresses.

Table. S9. The log2FC values of paralogs in decreasing patterns under four stresses.

\section{Competing interests}

The authors declare that no competing interests exist. 


\section{Funding}

This work was supported by the National Natural Science Foundation of China (Grant. 61501392, U1604112, 31701740), Nanhu Scholars Program for Young Scholars of XYNU.

\section{Authors' contributions}

SL and $Y Z$ implemented the algorithms and carried out the experiments. SL and LC drafted the manuscript. SL, YZ, ZZ and LC designed the study and analyzed the results. LC, and AG participated in the analysis and discussion. SL and TL contributed equally. All authors read and approved the final manuscript.

\section{Acknowledgments}

Authors thank anonymous reviewers for their comments on the manuscript. The linguistic editing and proofreading provided by TopEdit LLC during the preparation of this manuscript are acknowledged.

\section{Authors' information}

${ }^{1}$ College of Physics and Electronic Engineering, Xinyang Normal University, Xinyang, China.

${ }^{2}$ College of Life Sciences, Xinyang Normal University, Xinyang, China.

\section{References}

[1] Zhikai Liang, James C. Schnable. Functional Divergence between Subgenomes and Gene Pairs after Whole Genome Duplications. Molecular Plant,2018,11(3). DOI: 10.1016/j.molp.2017.12.010.

[2] Ren Ren, Haifeng Wang, Chunce Guo, Ning Zhang, Liping Zeng, Yamao Chen, Hong Ma, Ji Qi. Widespread Whole Genome Duplications Contribute to Genome Complexity and Species Diversity in Angiosperms. Molecular Plant,2018,11(3): 414-428. DOI: 10.1016/j.molp.2018.01.002.

[3] Christenhusz M J M, Byng J W. The number of known plants species in the world and its annual increase. Phytotaxa, 2016, 261(3):201. DOI: 10.11646/phytotaxa.261.3.1.

[4] Barker Michael S, Vogel Heiko, Schranz M Eric. Paleopolyploidy in the Brassicales: analyses of the Cleome transcriptome elucidate the history of genome duplications in Arabidopsis and other Brassicales. Genome Biology and Evolution,2009,1(1):391-399. DOI: 10.1093/gbe/evp040.

[5] Anne Roulin, Paul L. Auer, Marc Libault, Jessica Schlueter, Andrew Farmer, Greg May, Gary Stacey, Rebecca W. Doerge, Scott A. Jackson. The fate of duplicated genes in a polyploid plant genome. The Plant Journal,2013,73(1):143-153. DOI: 10.1111/tpj.12026. 
[6] Matthew J. Hegarty, Simon J. Hiscock. Genomic Clues to the Evolutionary Success of Polyploid Plants. Current Biology,2008,18(10). DOI: 10.1016/j.cub.2008.03.043.

[7] Marie Sémon, Kenneth H. Wolfe. Reciprocal gene loss between Tetraodon and zebrafish after whole genome duplication in their ancestor. Trends in Genetics,2007,23(3):108-112. DOI:

10.1016/j.tig.2007.01.003.

[8] Jiao Yuannian, Wickett Norman J, Ayyampalayam Saravanaraj, Chanderbali André S, Landherr Lena, Ralph Paula E, Tomsho Lynn P, Hu Yi, Liang Haiying, Soltis Pamela S, Soltis Douglas E, Clifton Sandra W, Schlarbaum Scott E, Schuster Stephan C, Ma Hong, Leebens-Mack Jim, dePamphilis Claude W. Ancestral polyploidy in seed plants and angiosperms. Nature,2011,473(7345):97-100. DOI: 10.1038/nature09916.

[9] Yang Zhenzhen, Wafula Eric K, Honaas Loren A, Zhang Huiting, Das Malay, Fernandez-Aparicio Monica, Huang Kan, Bandaranayake Pradeepa C G, Wu Biao, Der Joshua P, Clarke Christopher R, Ralph Paula E, Landherr Lena, Altman Naomi S, Timko Michael P, Yoder John I, Westwood James H, dePamphilis Claude W. Comparative transcriptome analyses reveal core parasitism genes and suggest gene duplication and repurposing as sources of structural novelty. Molecular biology and evolution,2015,32(3):767-790. DOI: 10.1093/molbev/msu343.

[10] Ning Zhang, Liping Zeng, Hongyan Shan, Hong Ma. Highly conserved low-copy nuclear genes as effective markers for phylogenetic analyses in angiosperms. New Phytologist,2012,195(4):923-937. DOI: 10.1111/j.1469-8137.2012.04212.x.

[11] Lynch M, Conery J S. The evolutionary fate and consequences of duplicate genes. Science,2000,290(5494):1151-1155. DOI: 10.1126/science.290.5494.1151.

[12] Yupeng W, Xiyin W, Haibao T, Xu T, Ficklin S. P, Alex F. F. Modes of Gene Duplication Contribute Differently to Genetic Novelty and Redundancy, but Show Parallels across Divergent Angiosperms. PLoS ONE, 2011, 6(12): e28150-. DOI: 10.1371/journal.pone.0028150.

[13] Gout Jean-Francois, Lynch Michael. Maintenance and Loss of Duplicated Genes by Dosage Subfunctionalization. Molecular biology and evolution,2015,32(8):2141-2148. DOI: 10.1093/molbev/msv095.

[14] Gu Z, Rifkin S A, White K P, Li W H. Duplicate genes increase gene expression diversity within and between species. Nature Genetics, 2004, 36(6):577-579. DOI: 10.1038/ng1355.

[15] Lynch Michael. Genomics. Gene duplication and evolution. Science,2002,297(5583):1551. DOI: 10.1126/science.293.5535.1551a.

[16] Innan Hideki, Kondrashov Fyodor. The evolution of gene duplications: classifying and distinguishing between models. Nature Reviews. Genetics,2010,11(2). DOI: 10.1038/nrg2689. 
[17] Yupeng Wang, Xiyin Wang, Andrew H Paterson. Genome and gene duplications and gene expression divergence: a view from plants. Annals of the New York Academy of Sciences,2012,1256(1):1-14. DOI: 10.1111/j.1749-6632.2011.06384.x.

[18] Khan Nadeem, Hu Chun-Mei, Amjad Khan Waleed, Naseri Emal, Ke Han, Huijie Dong, Hou Xilin. Evolution and Expression Divergence of E2 Gene Family under Multiple Abiotic and Phytohormones Stresses in Brassica rapa. BioMed research international,2018,2018. DOI: 10.1155/2018/5206758.

[19] Hodgins Kathryn A, Yeaman Sam, Nurkowski Kristin A, Rieseberg Loren H, Aitken Sally N. Expression Divergence Is Correlated with Sequence Evolution but Not Positive Selection in Conifers. Molecular biology and evolution,2016,33(6):1502-1516. DOI: 10.1093/molbev/msw032.

[20] Echave Julian, Wilke Claus O. Biophysical Models of Protein Evolution: Understanding the Patterns of Evolutionary Sequence Divergence. Annual review of biophysics,2017,46. DOI: 10.1146/annurev-biophys070816-033819.

[21] Dahai Gao, Dennis C. Ko, Xinmin Tian, Guang Yang, Liuyang Wang. Expression Divergence of Duplicate Genes in the Protein Kinase Superfamily in Pacific Oyster. Evolutionary Bioinformatics,2015,2015(Suppl. 1):57-65. DOI: 10.4137/EBO.S30230.

[22] Warnefors Maria, Kaessmann Henrik. Evolution of the correlation between expression divergence and protein divergence in mammals. Genome biology and evolution,2013,5(7):1324-1335. DOI: 10.1093/gbe/evt093.

[23] Moyers B T, Rieseberg L H. Divergence in Gene Expression Is Uncoupled from Divergence in Coding Sequence in a Secondarily Woody Sunflower. International Journal of Plant Sciences, 2013, 174(7):10791089. DOI: $10.1086 / 671197$.

[24] Severin Uebbing, Axel Künstner, Hannu Mäkinen, Niclas Backström, Paulina Bolivar, Reto Burri, Ludovic Dutoit, Carina F. Mugal, Alexander Nater, Bronwen Aken, Paul Flicek, Fergal J. Martin, Stephen M. J. Searle, Hans Ellegren. Divergence in gene expression within and between two closely related flycatcher species. Molecular Ecology,2016,25(9):2015-2028. DOI: 10.1111/mec.13596.

[25] Eduardo P.C. Rocha. The quest for the universals of protein evolution. Trends in Genetics,2006,22(8):412-416. DOI: 10.1016/j.tig.2006.06.004.

[26] Nuzhdin, S. V. Common Pattern of Evolution of Gene Expression Level and Protein Sequence in Drosophila. Molecular Biology and Evolution, 2004, 21(7):1308-1317. DOI: 10.1093/molbev/msh128.

[27] Liao, B.-Y. Low Rates of Expression Profile Divergence in Highly Expressed Genes and Tissue-Specific Genes During Mammalian Evolution. Molecular Biology and Evolution, 2006, 23(6):1119-1128. DOI: 10.1093/molbev/msj119. 
[28] Ostlund Gabriel, Schmitt Thomas, Forslund Kristoffer, Köstler Tina, Messina David N, Roopra Sanjit, Frings Oliver, Sonnhammer Erik L L. InParanoid 7: new algorithms and tools for eukaryotic orthology analysis. Nucleic Acids Research,2009,38(Database i): D196-D203. DOI: 10.1093/nar/gkp931.

[29] Shuaibin Lian, Tianliang Liu, Shengli Jing, Hongyu Yuan, Zaibao Zhang and Lin Cheng. Intrachromosomal colocalization strengthens co-expression, co-modification and evolutionary conservation of neighboring genes. BMC Genomics, (2018), 19:455-. DOI: 10.1186/s12864-018-4844-1.

[30] Clark, R. M., Schweikert, G., Toomajian, C. Common Sequence Polymorphisms Shaping Genetic Diversity in Arabidopsis thaliana. Science, 2007, 317(5836):338-342. DOI: 10.1126/science.1138632.

[31] Ji-Hye Jang, Yun Shang, Hyun Kyung Kang, Sun Young Kim, Beg Hab Kim, Kyoung Hee Nam. Arabidopsis galactinol synthases 1 (AtGOLS1) negatively regulates seed germination. Plant Science,2018,267:94-101. DOI: 10.1016/j.plantsci.2017.11.010.

[32] Chen Jui-Hung, Jiang Han-Wei, Hsieh En-Jung, Chen Hsing-Yu, Chien Ching-Te, Hsieh Hsu-Liang, Lin Tsan-Piao. Drought and Salt Stress Tolerance of an Arabidopsis Glutathione S-Transferase U17 Knockout Mutant Are Attributed to the Combined Effect of Glutathione and Abscisic Acid1. Plant Physiology,2012,158(1):340-351. DOI: 10.1104/pp.111.181875.

[33] Lee S Y, Boon N J, Webb A. A. R, Tanaka R. J. Synergistic Activation of RD29A via Integration of Salinity Stress and Abscisic Acid in Arabidopsis thaliana. Plant \& Cell Physiology, 2016, 57(10):21472160. DOI: $10.1093 /$ pcp/pcw132.

[34] Mark T. Waters, Adrian Scaffidi, Yueming K. Sun, Gavin R. Flematti, Steven M. Smith. The karrikin response system of A rabidopsis. The Plant Journal,2014,79(4). DOI: 10.1111/tpj.12430.

[35] Sangmin Lee, Pil Joon Seo, Hyo-Jun Lee, Chung-Mo Park. A NAC transcription factor NTL4 promotes reactive oxygen species production during drought-induced leaf senescence in Arabidopsis. The Plant Journal,2012,70(5). DOI: 10.1111/j.1365-313X.2012.04932.x.

[36] Li J, Zhong R, Palva E T. WRKY70 and its homolog WRKY54 negatively modulate the cell wallassociated defenses to necrotrophic pathogens in Arabidopsis. Plos One, 2017, 12(8): e0183731. DOI: 10.1371/journal.pone.0183731.

[37] Mirabella Rossana, Rauwerda Han, Allmann Silke, Scala Alessandra, Spyropoulou Eleni A, de Vries Michel, Boersma Maaike R, Breit Timo M, Haring Michel A, Schuurink Robert C. WRKY40 and WRKY6 act downstream of the green leaf volatile E-2-hexenal in Arabidopsis. The Plant journal: for cell and molecular biology,2015,83(6):1082-1096. DOI: 10.1111/tpj.12953.

[38] Nakata Masaru, Mitsuda Nobutaka, Herde Marco, Koo Abraham J K, Moreno Javier E, Suzuki Kaoru, Howe Gregg A, Ohme-Takagi Masaru. A bHLH-type transcription factor, ABA-INDUCIBLE BHLH-TYPE 
TRANSCRIPTION FACTOR/JA-ASSOCIATED MYC2-LIKE1, acts as a repressor to negatively regulate jasmonate signaling in arabidopsis. The Plant cell,2013,25(5):1641-1656. DOI: 10.1105/tpc.113.111112.

[39] Mizuno Takeshi, Yamashino Takafumi. Comparative transcriptome of diurnally oscillating genes and hormone-responsive genes in Arabidopsis thaliana: insight into circadian clock-controlled daily responses to common ambient stresses in plants. Plant and Cell Physiology,2008,49(3):481-487. DOI:

10.1093/pcp/pcn008.

[40] Fatma Kaplan, Charles L. Guy. RNA interference of Arabidopsis beta-amylase8 prevents maltose accumulation upon cold shock and increases sensitivity of PSIl photochemical efficiency to freezing stress. The Plant Journal,2005,44(5):14. DOI: 10.1111/j.1365-313x.2005.02565.x.

[41] Huang Junli, Gu Min, Lai Zhibing, Fan Baofang, Shi Kai, Zhou Yan-Hong, Yu Jing-Quan, Chen Zhixiang. Functional Analysis of the Arabidopsis PAL Gene Family in Plant Growth, Development, and Response to Environmental Stress. Plant Physiology,2010,153(4):1526-1538. DOI:

10.1104/pp.110.157370.

[42] Cuevas, Juan C, López-Cobollo, Rosa, Alcázar, Rubén, Zarza, Xavier, Koncz, Csaba, Altabella, Teresa,Salinas, Julio, Tiburcio, Antonio F, Ferrando, Alejandro. Putrescine Is Involved in Arabidopsis Freezing Tolerance and Cold Acclimation by Regulating Abscisic Acid Levels in Response to Low Temperature. Plant Physiology,2008,148(3):1094-1105. DOI: 10.4161/psb.4.3.7861.

[43] Maekawa Shugo, Inada Noriko, Yasuda Shigetaka, Fukao Yoichiro, Fujiwara Masayuki, Sato Takeo, Yamaguchi Junji. The carbon/nitrogen regulator ARABIDOPSIS TOXICOS EN LEVADURA31 controls papilla formation in response to powdery mildew fungi penetration by interacting with SYNTAXIN OF PLANTS121 in Arabidopsis. Plant physiology,2014,164(2):879-887. DOI: 10.1104/pp.113.230995.

[44] Raksha S, Seonghee L, Laura O, Elison B. Two chloroplast-localized proteins: AtNHR2A and AtNHR2B, contribute to callose deposition during nonhost disease resistance in Arabidopsis. Molecular PlantMicrobe Interactions, 2018: MPMI-04-18-0094-R-. DOI: 10.1094/MPMI-04-18-0094-R.

[45] Abdel-Ghany Salah Esmat. Contribution of plastocyanin isoforms to photosynthesis and copper homeostasis in Arabidopsis thaliana grown at different copper regimes. Planta,2009,229(4):767-779. DOI: $10.2307 / 23390386$.

[46] Gao Huiling, Xie Wenxiang, Yang Changhong, Xu Jingyi, Li Jingjun, Wang Hua, Chen Xi, Huang ChaoFeng. NRAMP2, a trans-Golgi network-localized manganese transporter, is required for Arabidopsis root growth under manganese deficiency. The New phytologist,2018,217(1):179. DOI: 10.1111/nph.14783.

[47] Remy Estelle, Cabrito Tânia R, Baster Pawel, Batista Rita A, Teixeira Miguel C, Friml Jiri, Sá-Correia Isabel, Duque Paula. A major facilitator superfamily transporter plays a dual role in polar auxin transport and drought stress tolerance in Arabidopsis. The Plant cell,2013,25(3):901-926. DOI:

10.1105/tpc.113.110353.

Page 18/31 
[48] Consonni, Chiara, Bednarek, Pawel, Humphry, Matt, Francocci, Fedra, Ferrari, Simone, Harzen, Anne,van Themaat, Emiel Ver Loren, Panstruga, Ralph. Tryptophan-Derived Metabolites Are Required for Antifungal Defense in the Arabidopsis mlo2 Mutant. Plant Physiology,2010,152(3):1544-1561. DOI: $10.2307 / 25680756$.

[49] Michael Hartmann, Tatyana Zeier, Friederike Bernsdorff, Vanessa Reichel-Deland, Denis Kim, Michele Hohmann, Nicola Scholten, Stefan Schuck, Andrea Bräutigam, Torsten Hölzel, Christian Ganter, Jürgen Zeier. Flavin Monooxygenase-Generated N-Hydroxypipecolic Acid Is a Critical Element of Plant Systemic Immunity. Cell,2018,173(2). DOI: 10.1016/j.cell.2018.02.049.

[50] Yosuke Maruyama, Natsuko Yamoto, Yuya Suzuki, Yukako Chiba, Ken-ichi Yamazaki, Takeo Sato, Junji Yamaguchi. The Arabidopsis transcriptional repressor ERF9 participates in resistance against necrotrophic fungi. Plant Science,2013,213:79-87. DOI: 10.1016/j.plantsci.2013.08.008.

[51] Jeon Jin, Cho Chuloh, Lee Mi Rha, Van Binh Nguyen, Kim Jungmook. CYTOKININ RESPONSE FACTOR2 (CRF2) and CRF3 Regulate Lateral Root Development in Response to Cold Stress in Arabidopsis. The Plant cell,2016,28(8):1828. DOI: 10.1105/tpc.15.00909.

[52] Zwack Paul J, Compton Margaret A, Adams Cami I, Rashotte Aaron M. Cytokinin response factor 4 (CRF4) is induced by cold and involved in freezing tolerance. Plant cell reports,2016,35(3):573-584. DOI: 10.1007/s00299-015-1904-8.

[53] Tsai K J, Chou S J, Shih M C. Ethylene plays an essential role in the recovery of Arabidopsis during post-anaerobiosis reoxygenation. Plant, Cell \& Environment, 2014, 37(10). DOI: 10.1111/pce.12292.

[54] Wang Xiaoping, Liu Shanda, Tian Hainan, Wang Shucai, Chen Jin-Gui. The Small Ethylene Response Factor ERF96 is Involved in the Regulation of the Abscisic Acid Response in Arabidopsis. Frontiers in plant science,2015,6. DOI: 10.3389/fpls.2015.01064.

[55] Fan Min, Bai Ming-Yi, Kim Jung-Gun, Wang Tina, Oh Eunkyoo, Chen Lawrence, Park Chan Ho, Son Seung-Hyun, Kim Seong-Ki, Mudgett Mary Beth, Wang Zhi-Yong. The bHLH transcription factor HBI1 mediates the trade-off between growth and pathogen-associated molecular pattern-triggered immunity in Arabidopsis. The Plant cell,2014,26(2):828-841. DOI: 10.1105/tpc.113.121111.

[56] Eunkyoo O, Jia-Ying Z, Ming-Yi B, Augusto, A. R, Yu S, Zhi-Yong. Cell elongation is regulated through a central circuit of interacting transcription factors in the Arabidopsis hypocotyl. eLife, 2014, 3. DOI: 10.7554/eLife.03031.

[57] Frerigmann Henning, Berger Bettina, Gigolashvili Tamara. bHLH05 is an interaction partner of MYB51 and a novel regulator of glucosinolate biosynthesis in Arabidopsis. Plant physiology,2014,166(1):349369. DOI: $10.1104 / p p .114 .240887$. 
[58] Ming Yang. The FOUR LIPS (FLP) and MYB88 genes conditionally suppress the production of nonstomatal epidermal cells in Arabidopsis cotyledons. American Journal of Botany,2016,103(9):15591566. DOI: 10.3732/ajb.1600238.

[59] Yan D, Easwaran V, Chau V, Okamoto M, lerullo M, Kimura M. NIN-like protein 8 is a master regulator of nitrate-promoted seed germination in Arabidopsis. Nature Communications, 2016, 7:13179. DOI: 10.1038/ncomms13179.

[60] Sozzani Rosangela, Maggio Caterina, Giordo Roberta, Umana Elisabetta, Ascencio-lbañez Jose Trinidad, Hanley-Bowdoin Linda, Bergounioux Catherine, Cella Rino, Albani Diego. The E2FD/DEL2 factor is a component of a regulatory network controlling cell proliferation and development in Arabidopsis. Plant Molecular Biology,2010,72(4-5):381-395. DOI: 10.1007/s11103-009-9577-8.

[61] Jiang Jianjun, Zhang Chi, Wang Xuelu. A recently evolved isoform of the transcription factor BES1 promotes brassinosteroid signaling and development in Arabidopsis thaliana. The Plant cell,2015,27(2). DOI: $10.1105 /$ tpc.114.133678.

[62] Liao X, Bao H, Meng Y, Plastow G, Moore S. Sequence, Structural and Expression Divergence of Duplicate Genes in the Bovine Genome. Plos One, 2014, 9(7): e102868. DOI:

10.1371/journal.pone.0102868.

[63] Wen-Hsiung Li, Jing Yang, Xun Gu. Expression divergence between duplicate genes. Trends in Genetics,2005,21(11):602-607. DOI: 10.1016/j.tig.2005.08.006.

[64] Zeng L, Zhang Q, Sun R, Kong H, Zhang N, Ma H. Resolution of deep angiosperm phylogeny using conserved nuclear genes and estimates of early divergence times. Nature Communications, 2014, 5:4956. DOI: $10.1038 /$ ncomms5956.

[65] Jiménez-Delgado Senda, Pascual-Anaya Juan, Garcia-Fernàndez Jordi. Implications of duplicated cis-regulatory elements in the evolution of metazoans: the DDI model or how simplicity begets novelty. Briefings in functional genomics \& proteomics,2009,8(4):266-275. DOI: 10.1093/bfgp/elp029.

[66] Zhong Zhenhui, Lin Lianyu, Chen Meilian, Lin Lili, Chen Xiaofeng, Lin Yahong, Chen Xi, Wang Zonghua, Norvienyeku Justice, Zheng Huakun. Expression Divergence as an Evolutionary Alternative Mechanism Adopted by Two Rice Subspecies Against Rice Blast Infection. Rice (New York, N.Y.),2019,12(1). DOI: 10.1186/s12284-019-0270-5.

[67] Vijayakumar G, Shaherin B, Prasannavenkatesh D, Sangdun C, Uversky V. N. Molecular Evolution and Structural Features of IRAK Family Members. PLoS ONE, 2012, 7(11): e49771-. DOI:

10.1371/journal.pone.0049771.

[68] Lehti-Shiu, Melissa D, Zou, Cheng, Hanada, Kousuke, Shiu, Shin-Han. Evolutionary History and Stress Regulation of Plant Receptor-Like Kinase/Pelle Genes. Plant Physiology,2009,150(1):12-26. DOI: 
[69] Hanada K, Kuromori T, Myouga F, Toyoda T, Shinozaki K, Walsh B. Increased Expression and Protein Divergence in Duplicate Genes Is Associated with Morphological Diversification. PLoS Genetics, 2009, 5(12): e1000781. DOI: 10.1371/journal.pgen.1000781.

[70] Silvia Coolen, Silvia Proietti, Richard Hickman, Nelson H. Davila Olivas, Ping-Ping Huang, Marcel C. Van Verk, Johan A. Van Pelt, Alexander H.J. Wittenberg, Martin De Vos, Marcel Prins, Joop J.A. Van Loon, Mark G.M. Aarts, Marcel Dicke, Corné M.J. Pieterse, Saskia C.M. Van Wees. Transcriptome dynamics of Arabidopsis during sequential biotic and abiotic stresses. The Plant Journal, 2016, 86(3):249-267. DOI: 10.1111/tpj.13167.

[71] Anders, S. Differential gene expression analysis based on the negative binomial distribution. Journal of Marine Technology \& Environment, 2009, 2(2).

[72] Langfelder P, Horvath S. WGCNA: an R package for weighted correlation network analysis. BMC Bioinformatics. 2008, 9(1):559. DOI: 10.1186/1471-2105-9-559.

[73] Yang Z H, Nielsen R, Goldman N, Pedersen A. M. K. Codon-Substitution Models for Heterogeneous Selection Pressure at Amino Acid Sites. Genetics, 2000, 155(1):431-449. DOI: 10.1002/1526968X(200005)27:1<32::AID-GENE50>3.0.CO;2-T.

[74] Lescot M, Déhais P, Thijs G, Marchal K, Moreau Y, Peer Y.V.D, Rouzé P, Rombauts S. PlantCARE, a database of plant cis-acting regulatory elements and a portal to tools for in silico analysis of promoter sequences. Nucleic Acids Res. 2002, 30, 325-327. DOI: 10.1093/nar/30.1.325.

[75] Crooks G.E, Hon G, Chandonia J.M, Brenner S.E. WebLogo: A sequence logo generator. Genome Res. 2004, 14, 1188-1190. DOI: 10.1101/gr.849004.

[76] Zhou Y, Zhou B, Pache L, Chang M, Khodabakhshi AH, Tanaseichuk O, Benner C, Chanda SK. 2019. Metascape provides a biologist-oriented resource for the analysis of systems-level datasets. Nat Commun. 10(1):1523. Doi: 10.1038/s41467-019-09234-6.

\section{Figures}


(A).
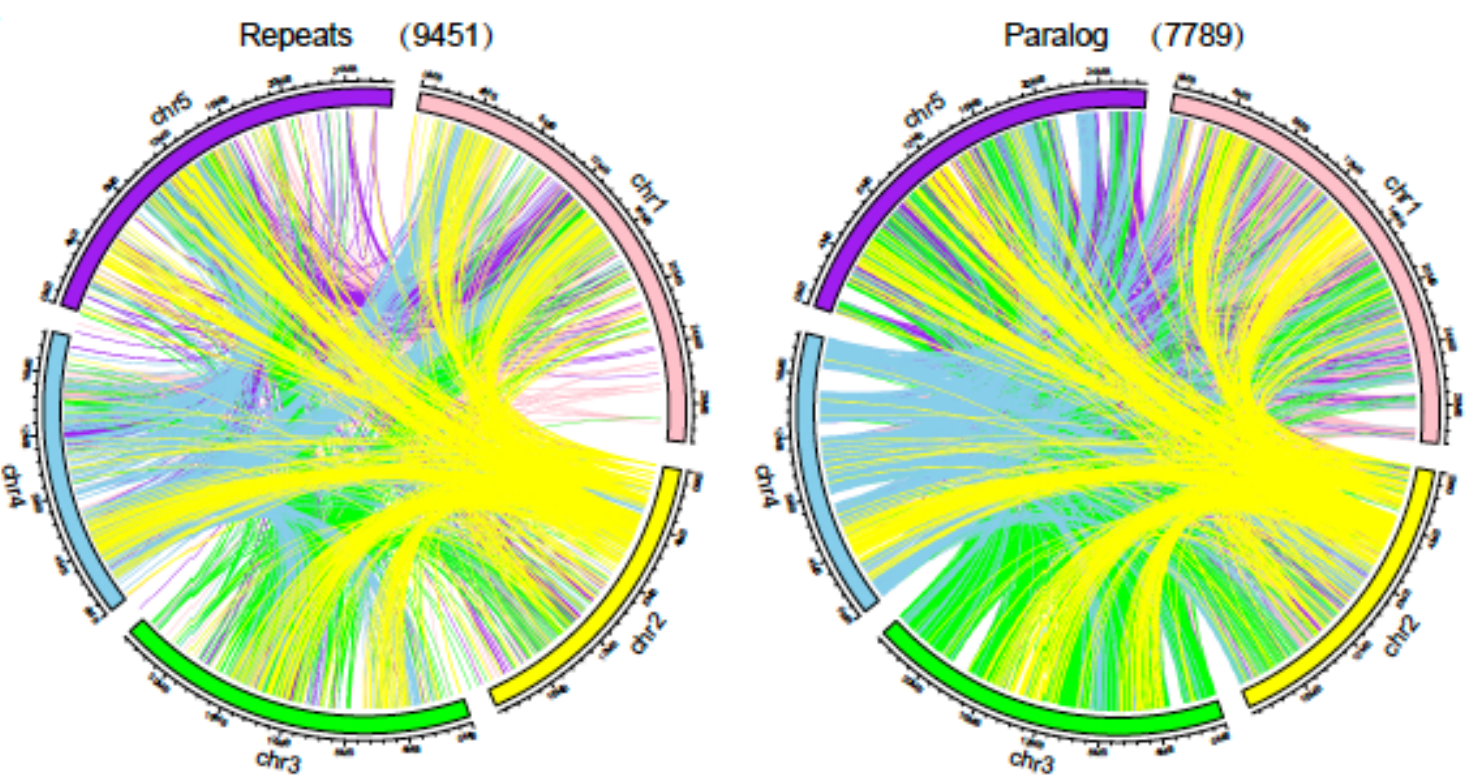

(B).
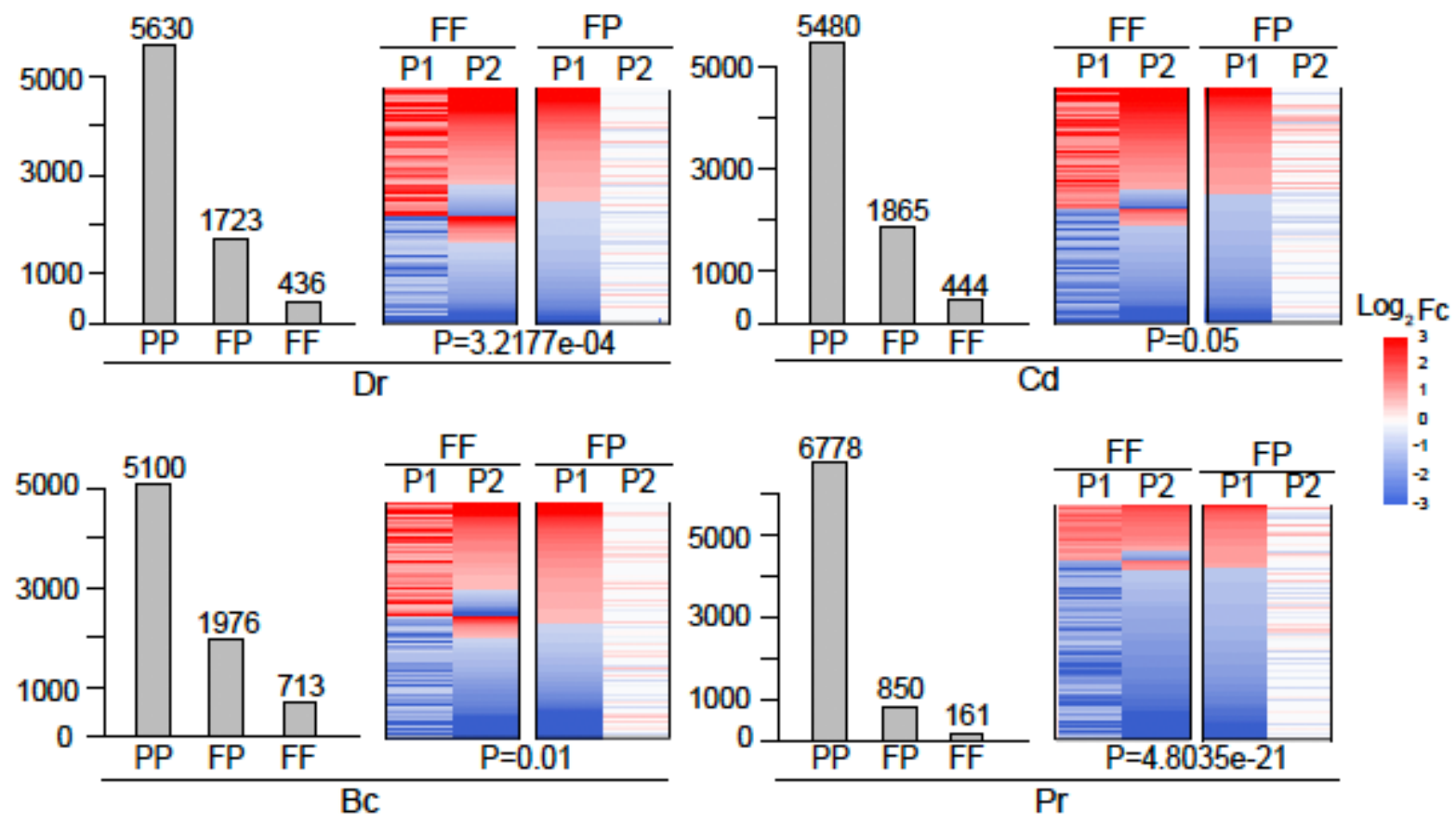

\section{Figure 1}

Distributions and expression classifications of the paralogs. (A) The distributions of 9,451 repetitive sequences and 7,789 paralogs in the chromosomes. (B) The identification and expression of the three types of paralogs under four different types of stress, namely, two biotic stresses (infection by the necrotrophic fungus Botrytis cinerea, $\mathrm{Bc}$; herbivory by the chewing larvae of Pieris rapae, $\mathrm{Pr}$ ) and two abiotic stresses (drought and cold). 
(A).

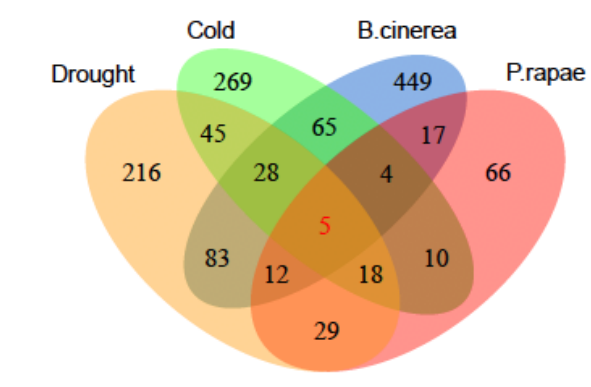

(B).

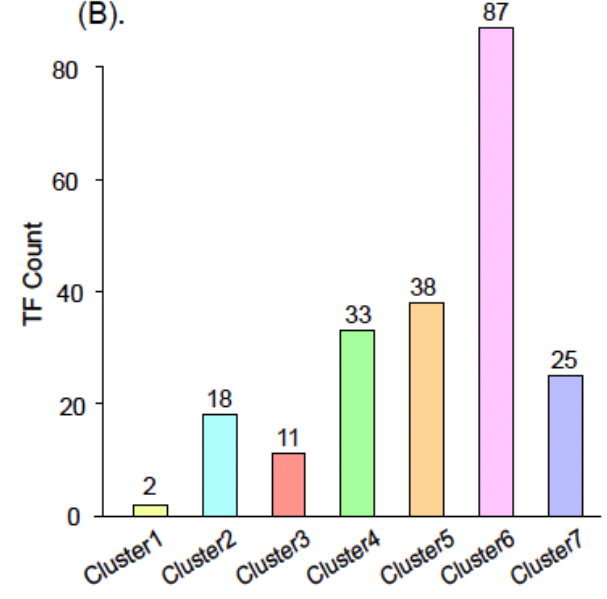

(C).

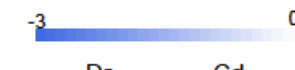

0

Cluster1
Cluster2
Cluster3
Cluster4

Cluster5
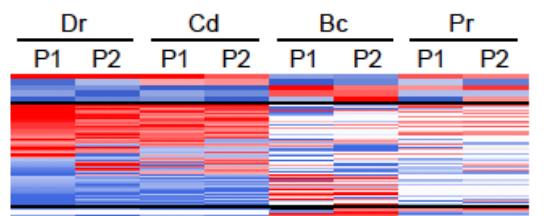

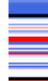

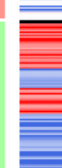

Cluster6

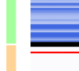

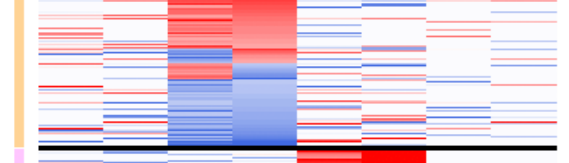

\section{in}

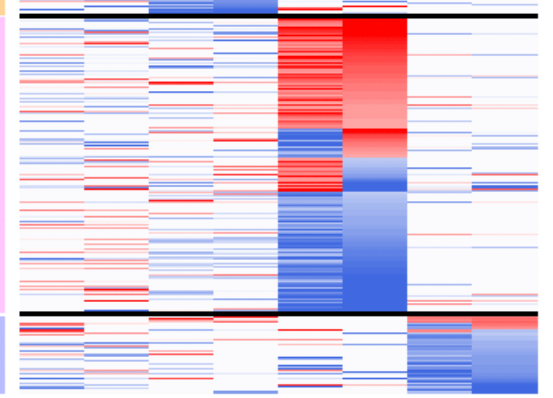

(D). \begin{tabular}{ll}
0 & $5 \quad 10$ \\
\hline
\end{tabular}

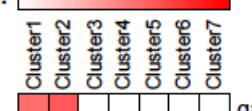

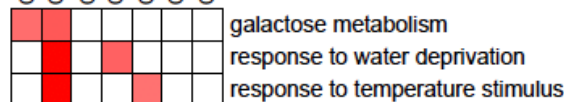
response to desiccation

response to wounding response to heat response to karrikin response to chitin response to drug response to fungus defense response to fungus defense response to bacterium response to bacterium immune system process response to oomycetes carbohydrate biosynthetic process photosynthesis drought recovery response to cold response to UV induced systemic resistance toxin metabolic process toxin catabolic process immune response response to toxic substance detoxification defense response to insect innate immune response

\section{Figure 2}

The differential expression patterns and function enrichment of the FF paralogs under four different types of stress. (A) The Venn diagram of the FF paralogs under four different types of stress. (B) The number of transcription factors in each cluster of FF paralogs. (C) The heatmap of seven expression modules of the FF paralogs under four different types of stress. Color bar represents the log2|FC| values, red represents up-regulation and blue represents down-regulation. (D) The functional enrichment of seven clustered paralogs in the heatmap. Color bar represents. 
(A).

Enhance

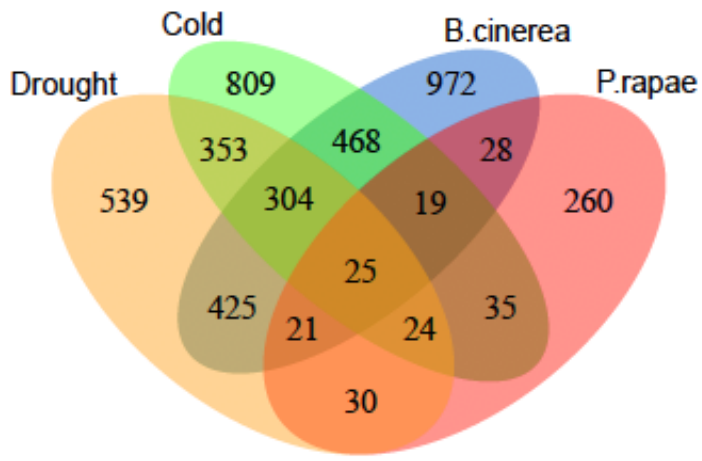

(C).
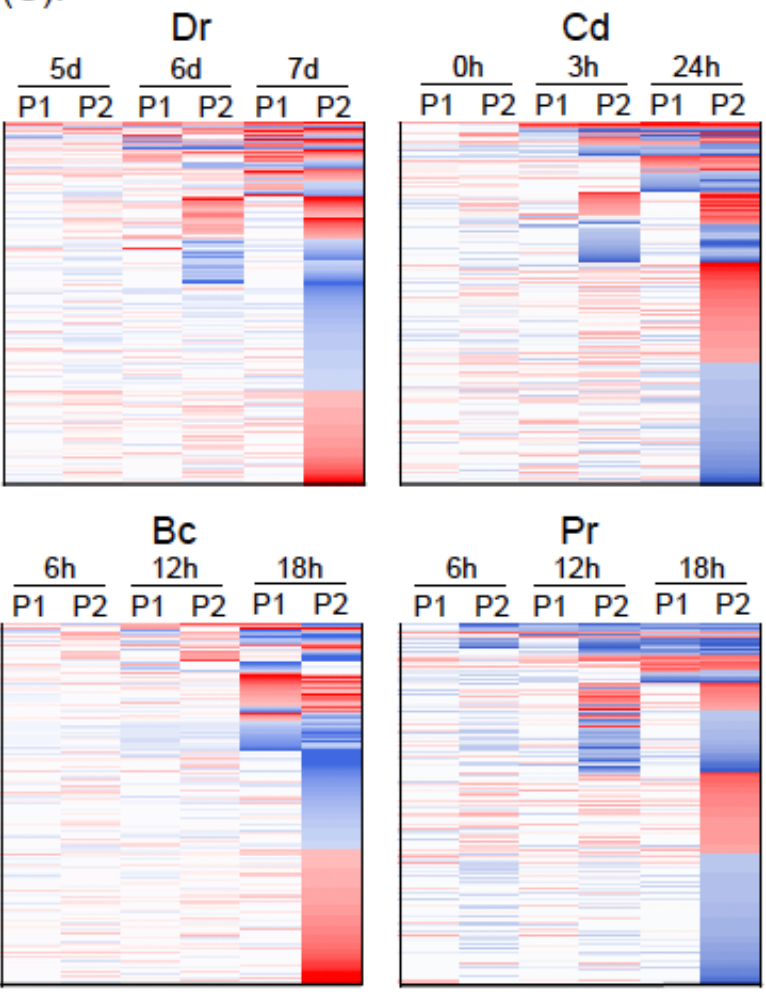

(B).

Decrease
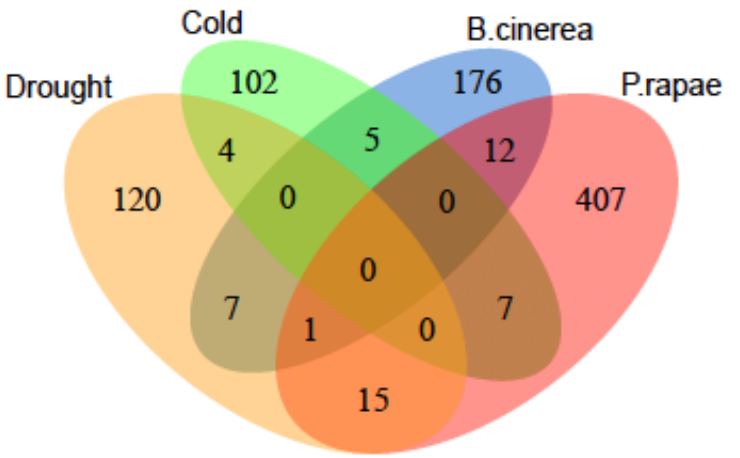

(D).

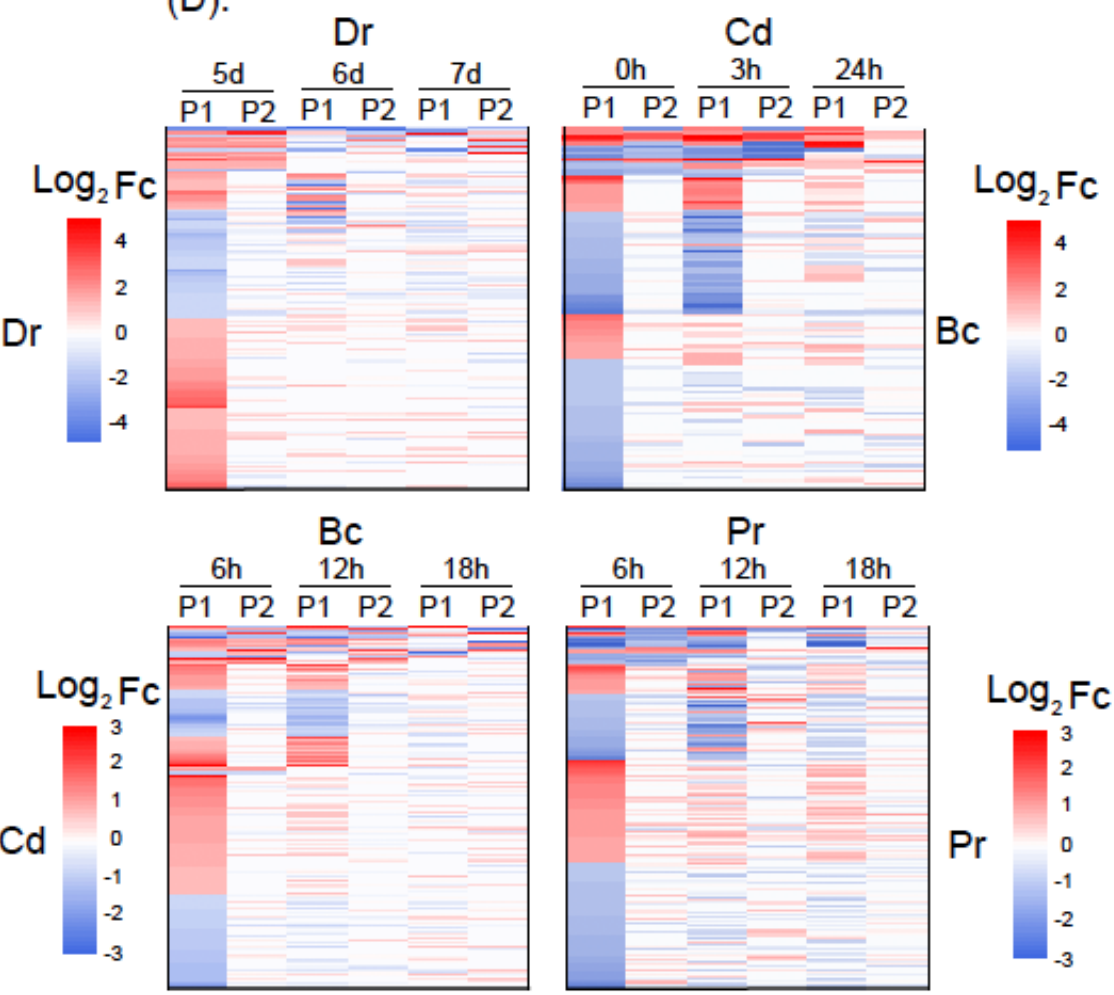

Figure 3

The expression pattern of enhancing and decreasing paralogs under different types of stress. (A) The Venn diagram of paralogs with enhancing expression patterns under four different types of stress. (B) The Venn diagram of paralogs with decreasing expression patterns under four different types of stress. (C) The heatmaps of paralogs with enhancing expression patterns under each stress condition. (D) The heatmaps of paralogs with decreasing expression patterns under each stress condition. 


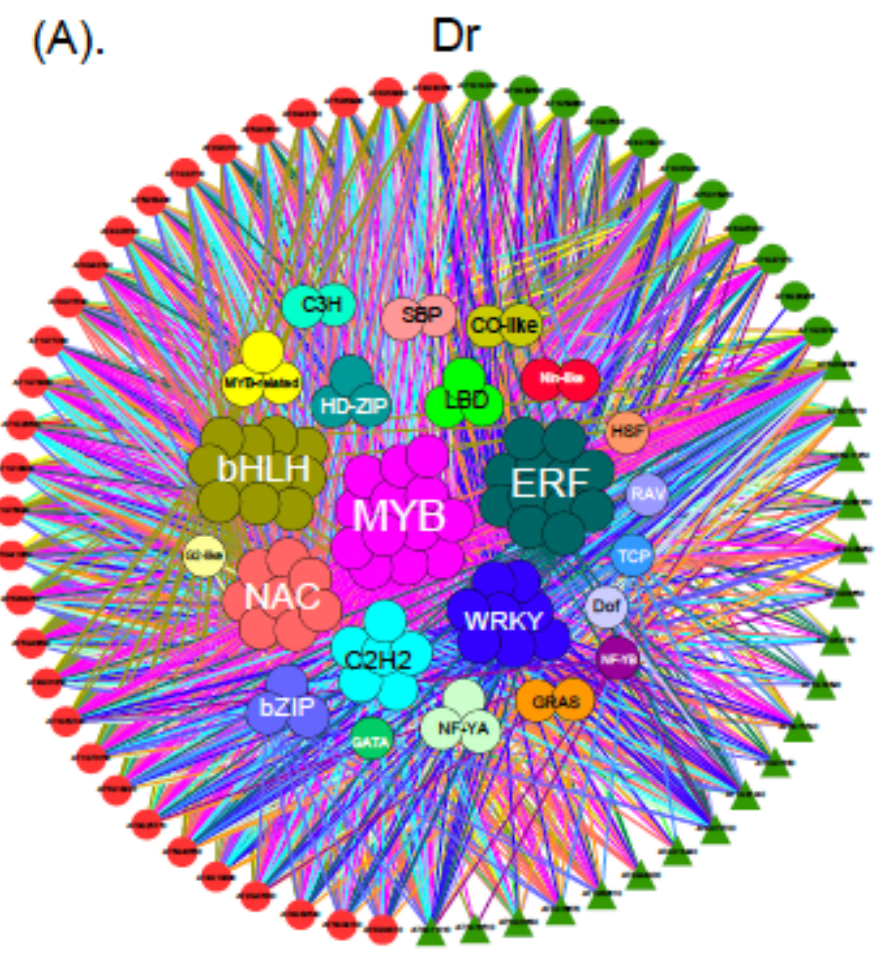

(B).

Cd

(C).

\section{$\mathrm{Bc}$}
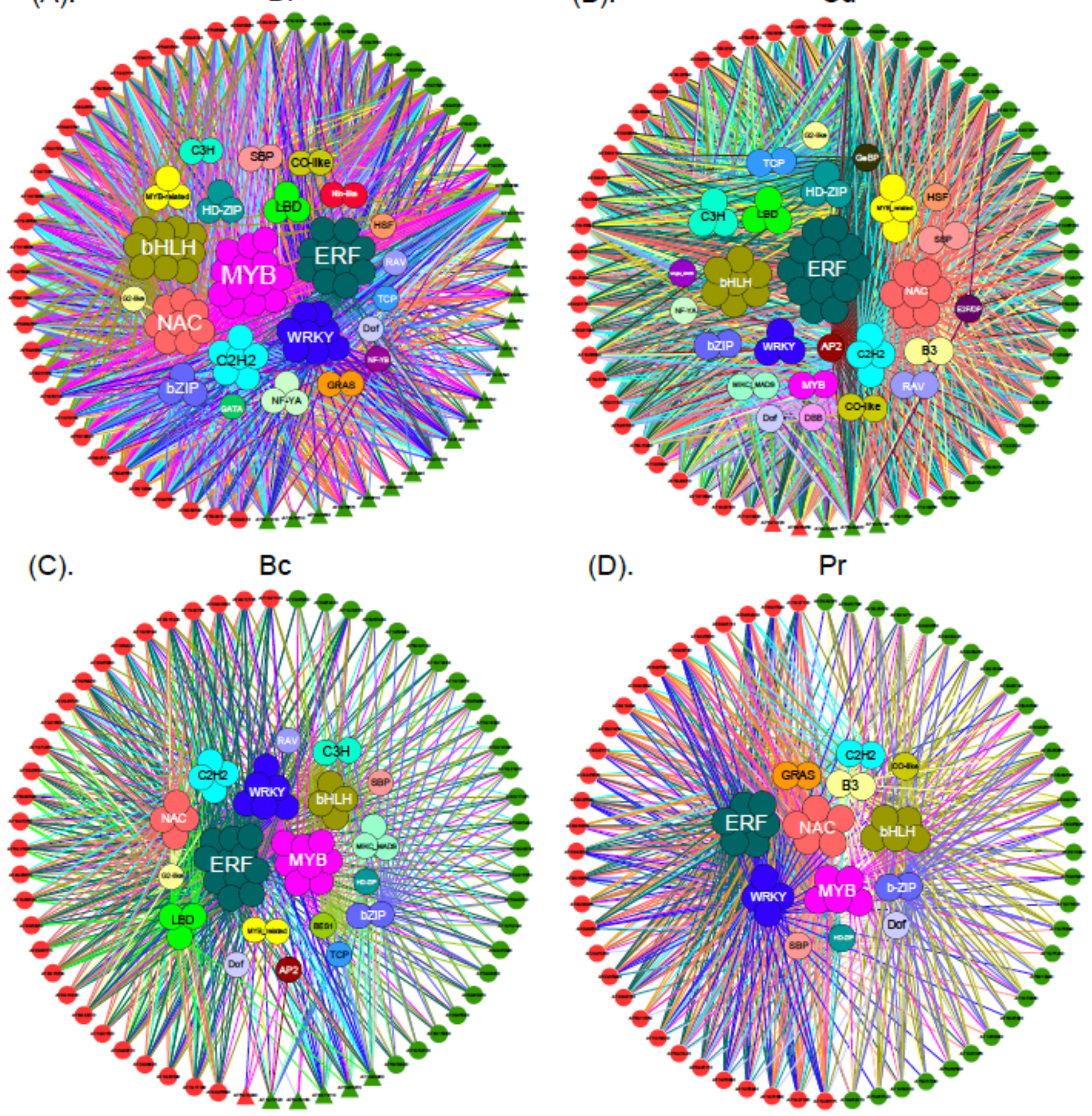

(D).

$\mathrm{Pr}$

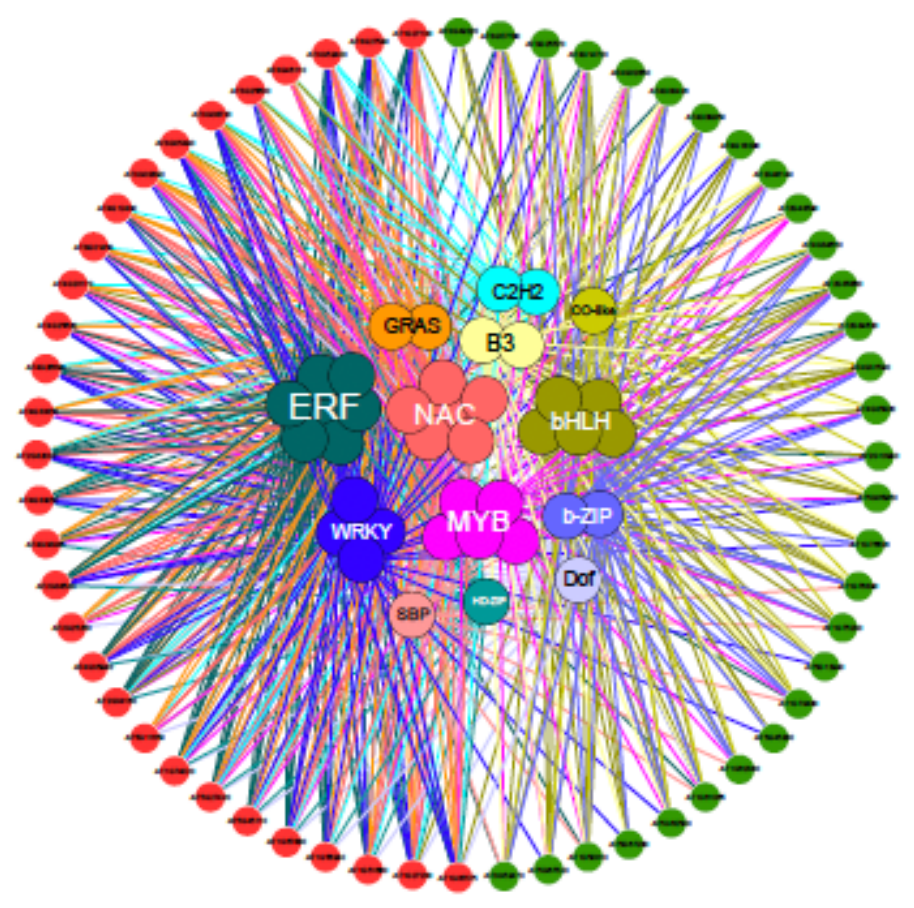

Figure 4

The co-expression networks of DEPs with enhancing and decreasing patterns under four different types of stress. The outer circle represents differentially expressed paralogs, red (left) and green (right) represent paralogs with enhancing and decreasing expression patterns, respectively. The triangle represents the up-regulated genes, the circle represents the down-regulated genes. The inner circle represents the co-expressed TFs. 

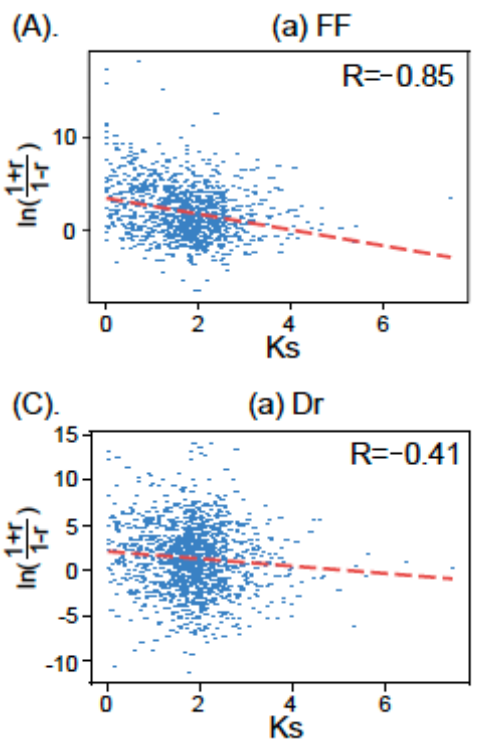

(c) Bc

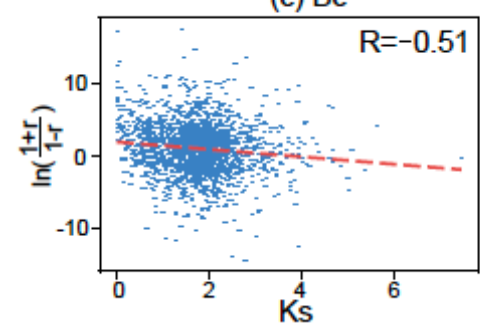

(b) FP

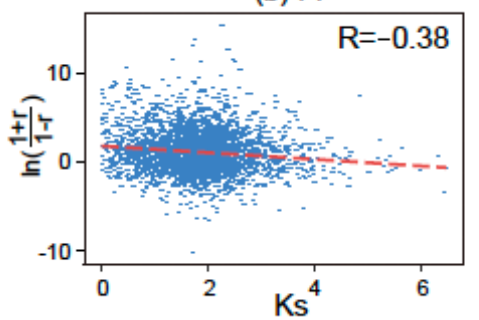

(b) Cd

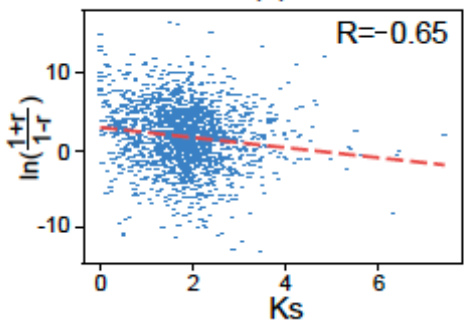

(d) $\mathrm{Pr}$

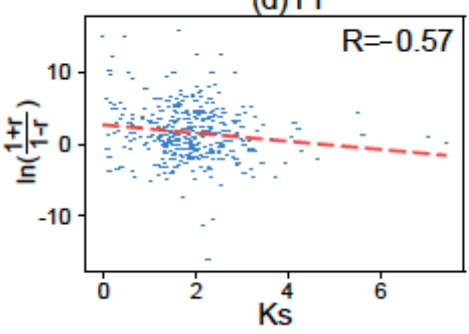

(B).

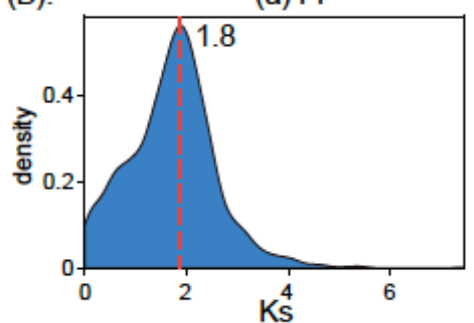

(a) $\mathrm{Dr}$

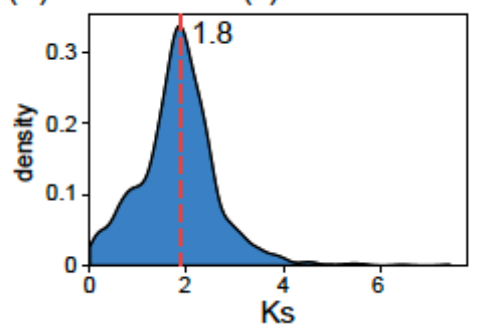

(c) $\mathrm{Bc}$

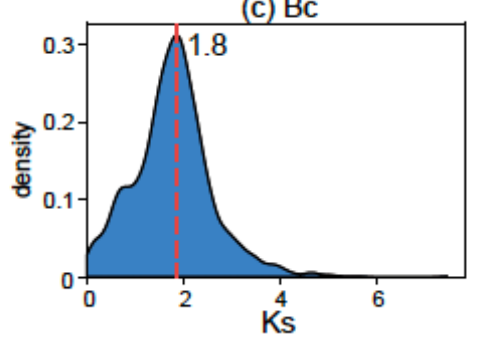

(b) FP

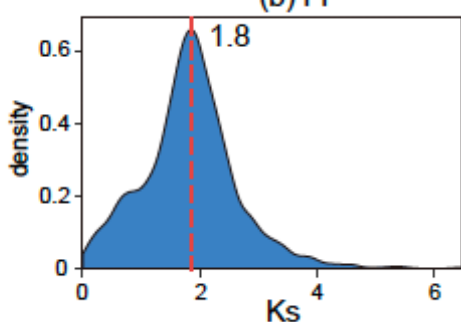

(b) Cd

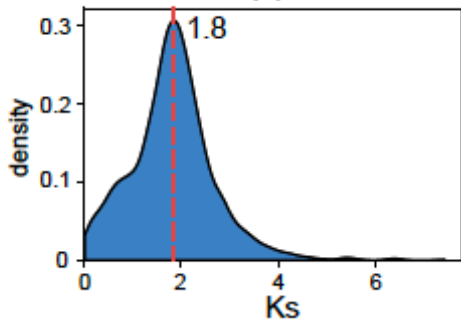

(d) $\mathrm{Pr}$

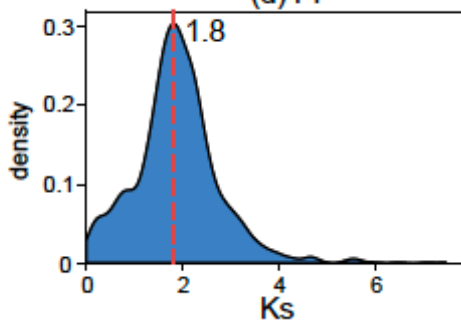

Figure 5

The regression results of the expression divergences and sequence divergences. (A) The regression results of FF (a) and FP (b) paralogs under all four types of stress, respectively. (B) The density plot of Ks values of FF (a) and FP (b) paralogs under all four types of stress, respectively. (C) The regression results of enhancing and decreasing expression paralogs under each stress condition, respectively. (a) $\mathrm{Dr}$, (b) Cd, (c) Bc, and (d) Pr. (D) The density plot of Ks values of enhancing and decreasing expression paralogs under each of the four types of stress, respectively. (a) Dr, (b) Cd, (c) Bc, and (d) Pr. 

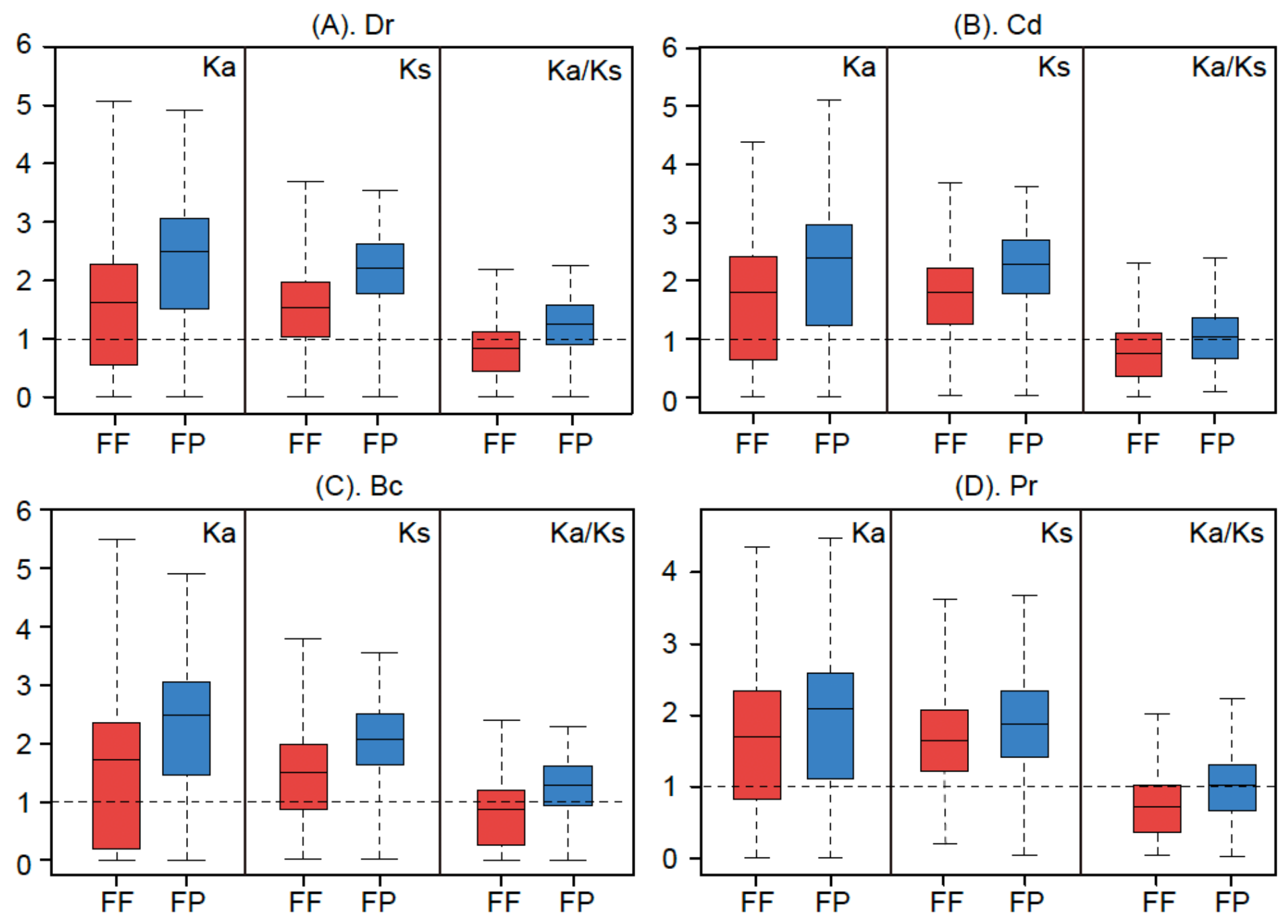

Figure 6

The boxplot of $\mathrm{Ka}$, Ks and $\mathrm{Ka} / \mathrm{Ks}$ of FF and FP DEPs under four different stress. 
(A).

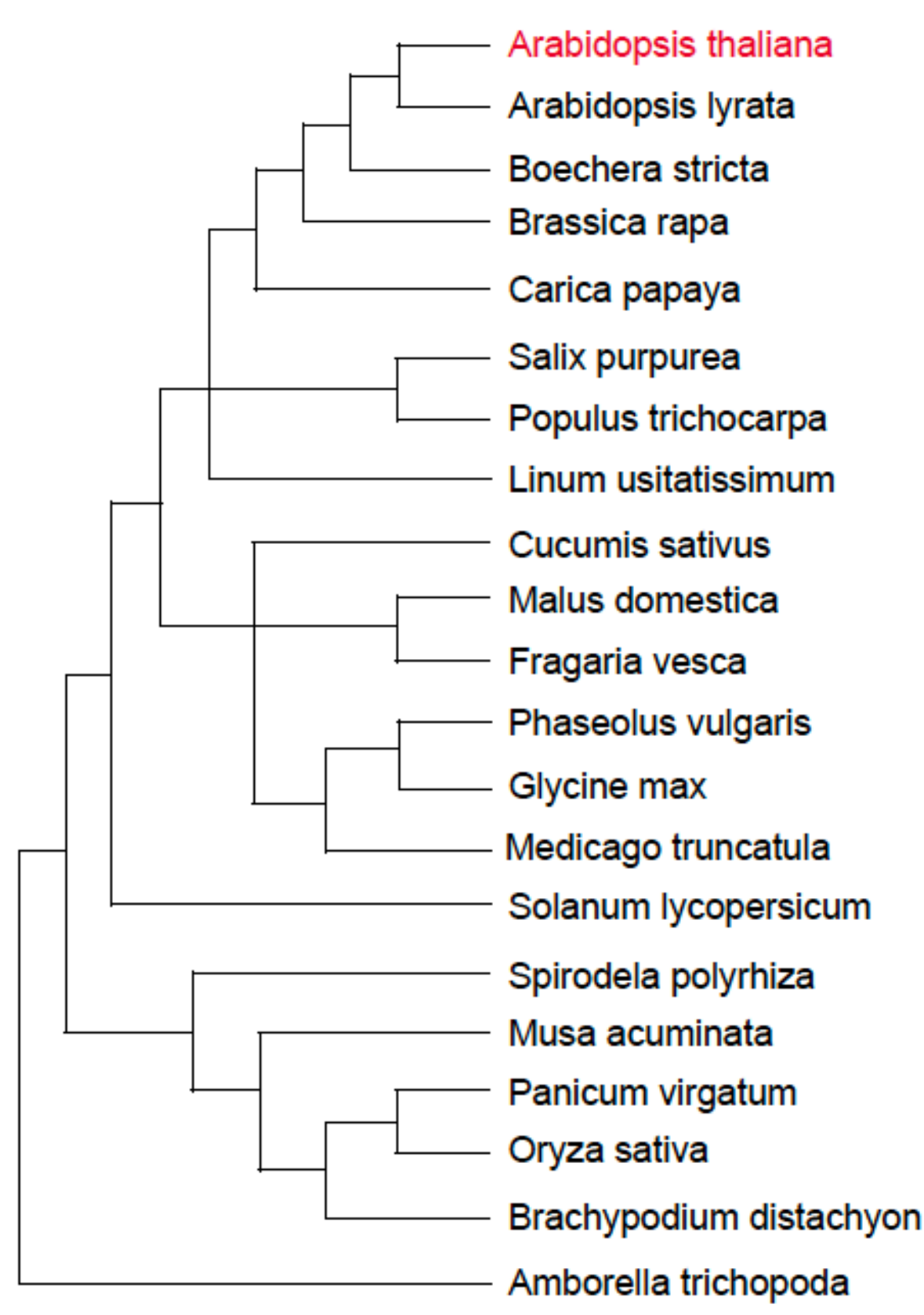

(B).

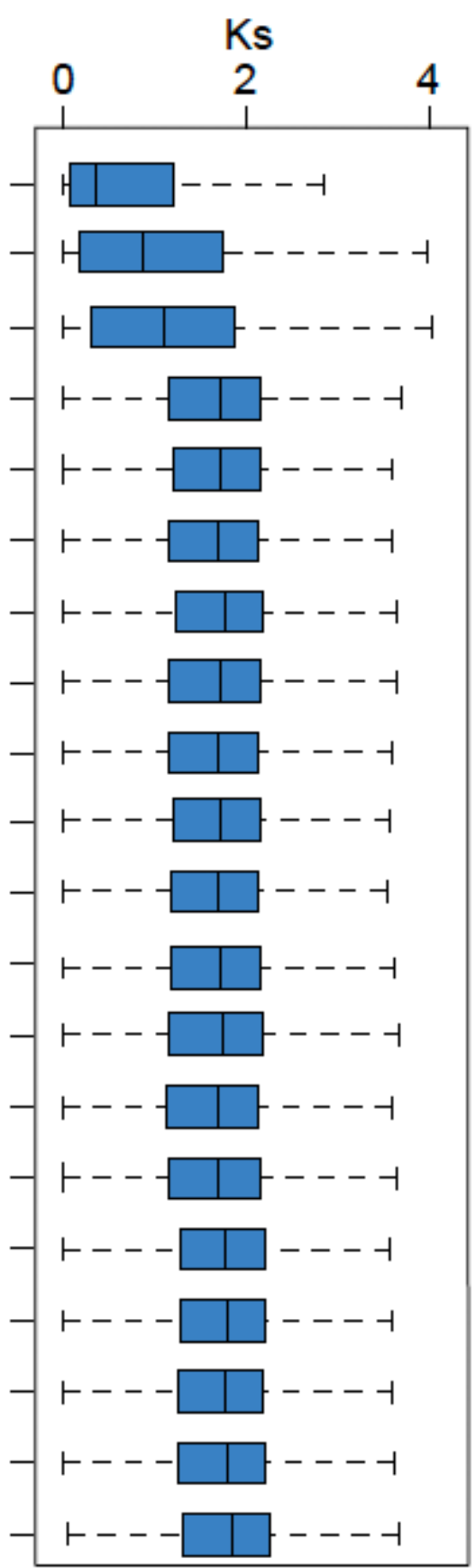

Figure 7

(A) The phylogenetic tree of the 21 species presented in this study. (B) The boxplot of the Ks values of the paralogs between Arabidopsis thaliana and the other 20 species. 


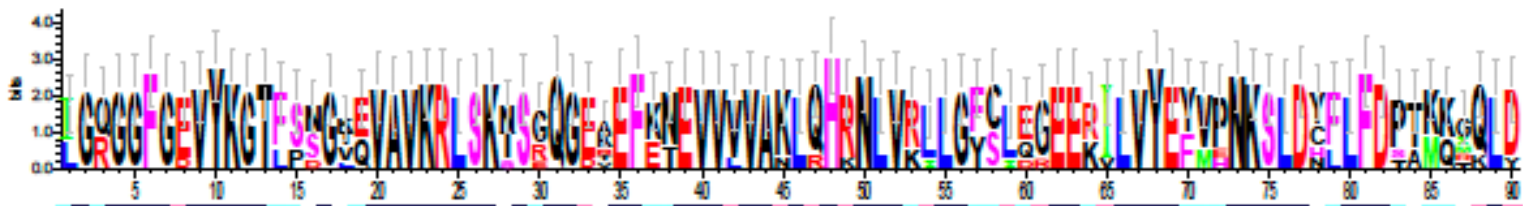

AT2023180 IGQGGF GEVYKGTLSD GTEVAVKRLSKSSGQGEVEFKNEVVLVAKL QHRNLVRLLGF CLDGEERVLVYEYVPNKSLDYFLFDPAKKGQLD 90 AT 023310 IGRGGF GEVYKGTF SNGKEVAVKRLSKNSRQGEAEFKTEVVVVAKL QHRNLVRLLGF SLQGEER ILVYEYMPNKSLDCLLFDPTKQIQLD 90 ATA23160 I GRGGF GEVYKGTF SNGKEVAVKRLSKNSRQGEAEFKTEVVVVAKL QHRNLVRLLGFSLQGEER ILVYEYMPNKSLDCLLFDPTKQTQLD 90 AT4023150 IGRGGF GDVYKGTF SNGT TV AVKRLSKTSEQGDTEF KNEVVVVANLRHKNLVRILGFSIEREER ILVYEYVENKSLDNFLFDPAKKGQLY 90

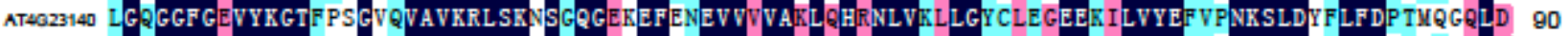

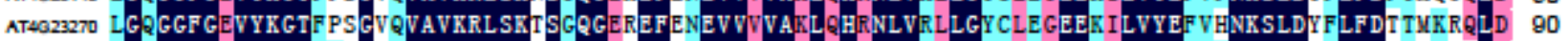
AT4023280 LGQGGF GEVYKGTLSSGLQVAVKRLSKTSGQGEKEFENEVVVVAKL QHRNLVKLLGYCLEGEEKILVYEFVPNKSLDHFLFDSTMKYKLD 90
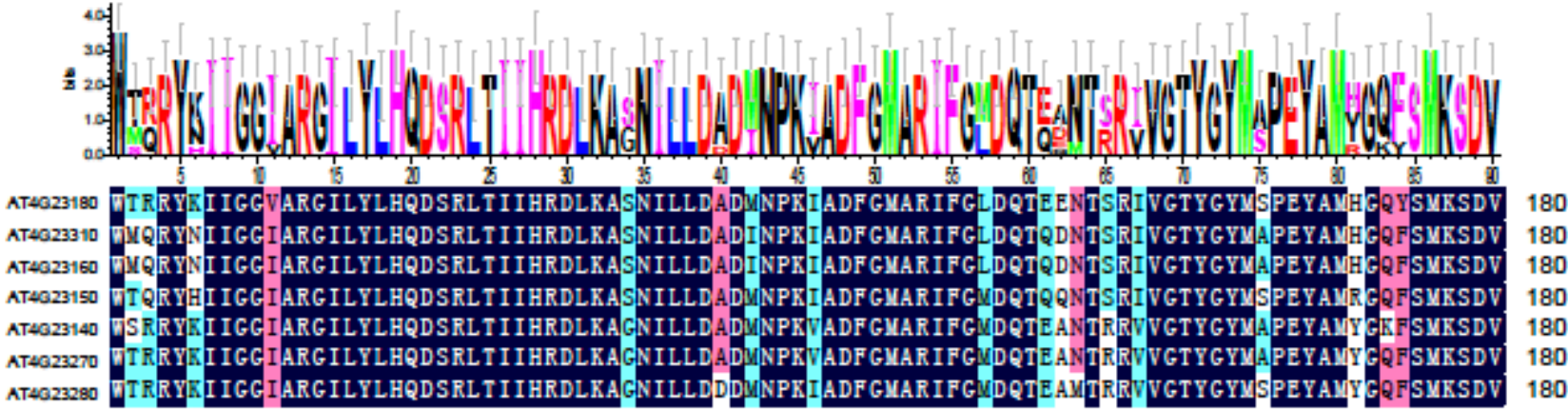

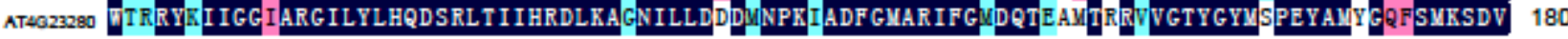
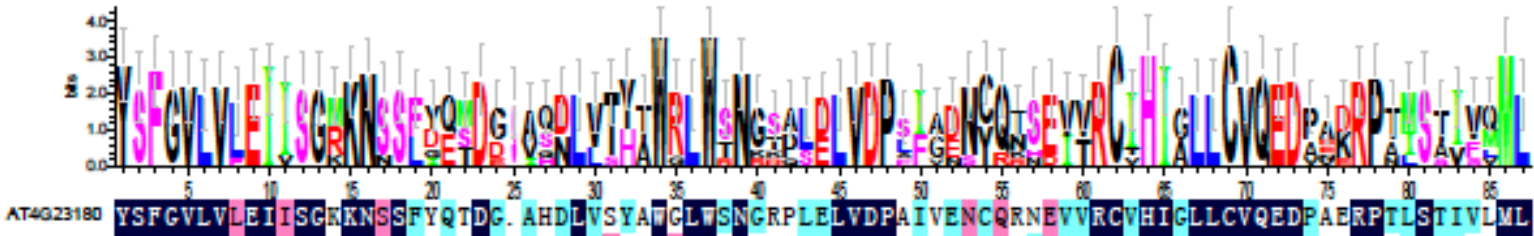

ATLO23310 YSF GVLVLE I ISGRINSSFGESDG. AQDLLTHAFRL FTNKKALDLVDPL I AENCQNSEVVRCIH I GLLCVQEDPARRPA ISTVFYYL

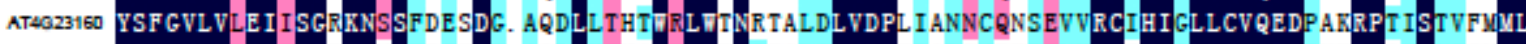
ATLO23150 YSF GVLVLE I I SGRKNNSF I ETDD. AQDLVTHAFRL TRNGTALDL VDPF I ADSCRRSEVVRCTH I GLLCVQEDPVRRPAMST ISVYL

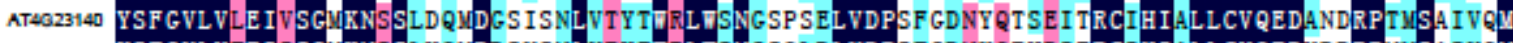

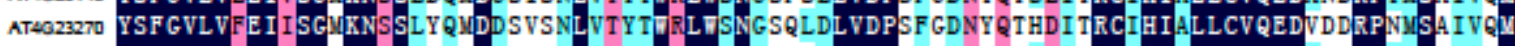

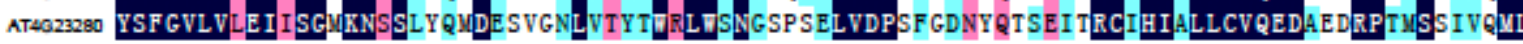

\section{Figure 8}

The conserved protein domain sequences of the paralogs in all 21 species. 
(A).

\begin{tabular}{|c|c|c|c|c|c|c|}
\hline Motif 1 & Motif 2 & Motif 3 & Motif 4 & Motif 5 & Motif 6 & Motif 7 \\
\hline Motif 9 & Motif 10 & Motif 11 & Motif 12 & Motif 13 & & \\
\hline
\end{tabular}

Name Motif location

\begin{tabular}{l} 
AT4G23180_14 \\
\hline
\end{tabular}

\begin{tabular}{|l|l|l|l|l|l|l|l|l|l|l|l|l|l|l|} 
AT4G23140 & 14 & 10 & 5 & 7 & 8 & 8 & 12 & 11 & 3 & 4 & 1 & 2 & 6 & 13 \\
\hline
\end{tabular}

\begin{tabular}{|l|l|l|l|l|l|l|l|l|l|l|l|l|l|l|l|l|l|l|} 
AT4G23160 & 14 & 10 & 5 & 7 & 8 & 8 & 12 & 11 & 3 & 4 & 1 & 2 & 6 & 13 & 15 \\
\hline
\end{tabular}

\begin{tabular}{|l|l|l|l|l|l|l|l|l|l|l|l|l|l|l|} 
AT4G23150 & 14 & 10 & 5 & 7 & 8 & 8 & 12 & 11 & 3 & 4 & 1 & 2 & 6 & 13 \\
\hline
\end{tabular}

\begin{tabular}{|l|l|l|l|l|l|l|l|l|l|l|l|l|l|l|l|l|l|} 
AT4G23280 & 14 & 10 & 5 & 7 & 8 & 8 & 12 & 11 & 3 & 4 & 1 & 2 & 6 & 13 & 15
\end{tabular}

\begin{tabular}{|l|l|l|l|l|l|l|l|l|l|l|l|l|l|l|l|l|l|l|l|} 
AT4G23310 & 14 & 10 & 5 & 10 & 5 & 7 & 8 & 8 & & 12 & 11 & 3 & 4 & 1 & 2 & 6 & 13 \\
\hline
\end{tabular}

\begin{tabular}{|l|l|l|l|l|l|l|l|l|l|l|l|l|l|l|} 
AT4G23270 & 10 & 5 & 7 & 8 & 8 & 12 & 11 & 3 & 4 & 1 & 2 & 6 & 13 & 15 \\
\hline
\end{tabular}

(B).

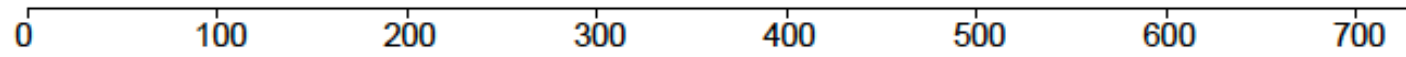

Name Logo

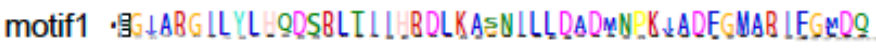

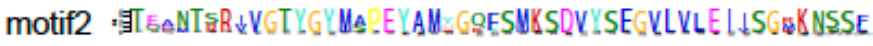

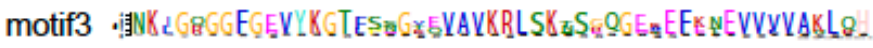
motif4 :

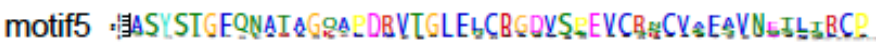

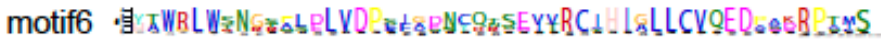
motif7 :

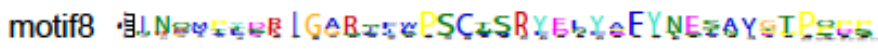

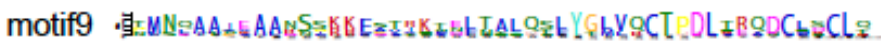

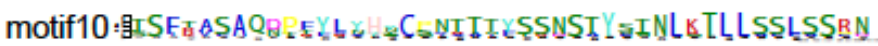
motif11 具

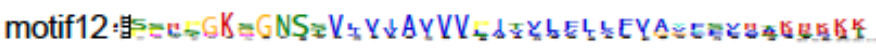

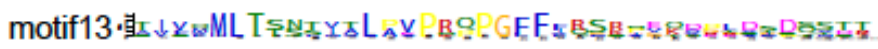

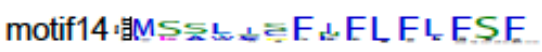

motif15:盺 $\downarrow$ DDes L.IDt

\begin{tabular}{|c|c|c|}
\hline Sequence & Width & TF \\
\hline GIARGILYLHQDSRLTIIHRDLKASNILLADMNPKIADFGMARIFGMDQ & 50 & LBD \\
\hline TZANTSRIVGTYGYMAPEYAMHGQFSMKSDVYSFGVLVLEIISGRKNSSF & 50 & ARF \\
\hline NKJGQGGFGEVYKGTFSNGVZVVAVKRLSKTSGQGEKEFINEVWVVAKLQH & 50 & SAP \\
\hline NLVRLLGFCLEGEERILVYEYVPNKSLDYFLFDPTKKGQLDWTRRYKIIG & 50 & Whirly \\
\hline ASYSTGFQNATAGQAPDRVTGLLLCRGDVSPEVCRNCVAFAVNETLTRCP & 50 & SRS \\
\hline YTWRLWSNGSPLDLVDPSIADNCQTSEYVRCIHIGLLCVQEDPADRPTMS & 50 & Whirly \\
\hline EAVFYYEZCILRYSNKNILSTLNTBGGVFMLNARNPISVKQ & 41 & \\
\hline INQMPYDRIGARIFWPSCTSRYELYYFYNESAVGTPPPP & 39 & Dof \\
\hline TMNQAAIEAANSSKKFATRKTELTALQSLYGLVQCTPDLTRCOCLSCLQ & 49 & GRAS \\
\hline TSFRASACDPFYLYHNCPNITTYSSNSTYSTNLKTLSSLSSRN & 44 & LBD \\
\hline AEDGDOITTADSLQLDYRAIQAATNDFLE & 29 & \\
\hline SPRPGKGGNSSVJVIAVWPITVLFLUFVAYFSVRAKRKKK & 41 & \\
\hline TIVQMLTSNTIALPVPRQPGFFFRSRPEKDPLDSDSSTT & 39 & Whirly \\
\hline MSSUISFIFLFLFSF & 15 & \\
\hline SIDDASITDLYPR & 15 & \\
\hline
\end{tabular}

\section{Figure 9}

The conserved motifs of the paralogs in all 21 species.

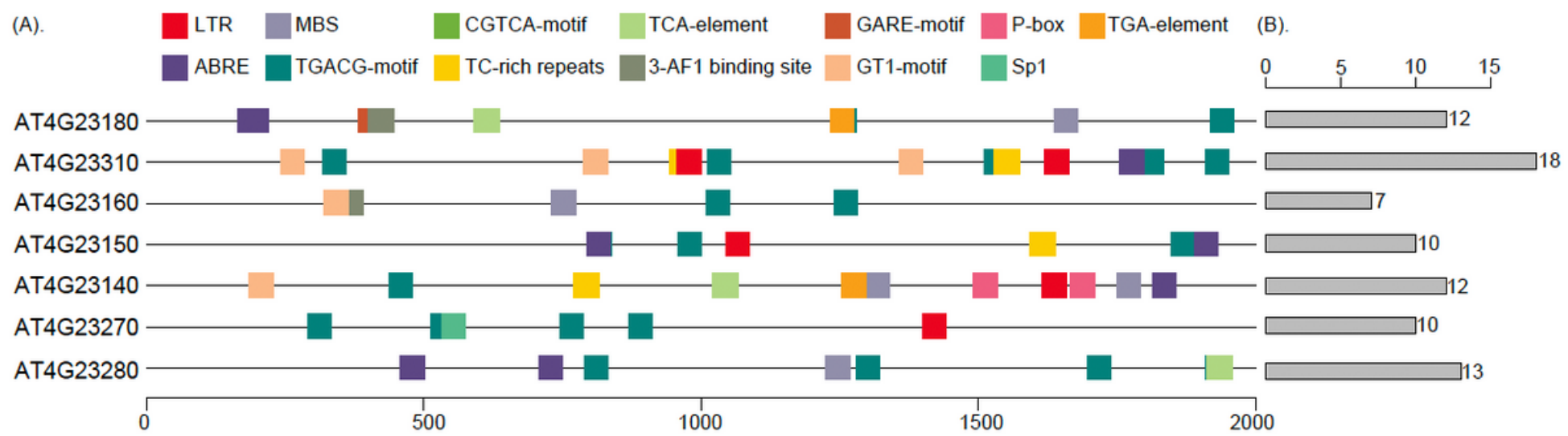

\section{Figure 10}

(A) The top ten cis-elements of STKc_IRAK in the 2000-bp promoter sequence. (B) The number of ciselements in each gene. 


\section{Supplementary Files}

This is a list of supplementary files associated with this preprint. Click to download.

- Fig.S3.pdf

- Fig.S2.pdf

- TableS1.Thelistof7789paralogs.xlsx

- TableS4.ThelistofFFFPandPPparalogs.xlsx

- TableS7.Thelog2FCvaluesofsevenFP.xIsx

- TableS9.Thelog2FCvaluesindecreascingpattern.xlsx

- TableS6.Thelog2FCvaluesofsevenFF.xlsx

- TableS3.Theinteractioninformationofrepeatss.xIsx

- TableS2.Theinteractioninformationofparalogs.xlsx

- Fig.S1.pdf

- TableS8.Thelog2FCvaluesinenhancingpatterns.xlsx

- TableS5.Thelog2FCvaluesofFFandFPparalogs.xIsx 